\title{
Maximum actuation strain for dissipative dielectric elastomers with simultaneous effect of pre-stretch and temperature
}

\author{
Lei Deng ${ }^{\mathrm{a}}$, Zhicheng $\mathrm{He}^{\mathrm{a}, \mathrm{b}^{*}}$, Eric $\mathrm{Li}^{\mathrm{c}^{*}}$, Shoue Chen ${ }^{\mathrm{a}}$ \\ ${ }^{\mathrm{a}}$ State Key Laboratory of Advanced Design and Manufacturing for Vehicle Body, Hunan University, \\ Changsha, 410082 P. R. China \\ ${ }^{b}$ Key Laboratory of Advanced Manufacture Technology for Automobile Parts (Chongqing University of \\ Technology), Ministry of Education \\ ${ }^{c}$ Department of Mechanical and Automation Engineering, The Chinese University of Hong Kong, \\ Shatin, NT, Hong Kong, China
}

\begin{abstract}
A dielectric elastomer can generate giant deformation by the voltage actuation, but the deformation is often hindered by the electromechanical instability (EMI) and 'snap-through deformation', which may lead to electrical breakdown (EB). In this study, for the first time, the mathematical model is established for dissipative dielectric elastomers in the dynamic model with simultaneous effect of pre-stretch and temperature in order to achieve maximum actuation strain. The deformation of the dissipative dielectric elastomer: VHB 4905/4910 is investigated for the two simple actuation methods: constant and ramping voltage actuation, respectively. The best combined conditions of voltage and pre-stretch to obtain a large deformation at different operating temperatures are studied in detail. Under the best combined conditions, the influences of three factors: voltage, pre-stretch and temperature on the maximum actuation strain are analyzed. This study should offer a great help in the design of dielectric elastomer actuators, and give the guidance to the accomplishment of the large deformation of dissipative dielectric elastomer actuators.
\end{abstract}

Key words: dissipative dielectric elastomers; maximum actuation strain; viscoelasticity; pre-stretch; temperature; electromechanical instability; snap-through deformation

\footnotetext{
* Corresponding author. Tel. /fax: +86 18607720701

E-mail address: hezhicheng815@gmail.com (Zhicheng He) ericsg2012@gmail.com (Eric Li)
} 


\section{Introduction}

Due to its advantages such as large deformation, fast response, low cost, lightweight, high energy density and low noise, dielectric elastomers have been widely used to make diverse practical applications such as artificial muscles, energy harvesters, soft robotics and braille displays[1-9]. Subject to a voltage across its thickness direction, a dielectric elastomer membrane reduces its thickness and expands in plane area, which is known as the electrical actuation [10-13]. For a dielectric elastomer used as an actuator, it is important to achieve a large actuation deformation induced by a voltage, which can improve its performance in the practical application.

However, the large actuation deformation is often hindered by the modes of instabilities during the actuation process. As the applied voltage across its thickness direction increases, the membrane may thin down drastically. Thus, a higher electric field will be induced by the same voltage. This positive feedback results in electromechanical instability (EMI) and 'snap-through deformation', which may lead to electrical breakdown (EB) [14-19]. The EMI is considered as the key factor that restricts the realization of a large deformation [16, 20, 21]. Recent research has revealed that large actuation strain over $100 \%$ is attainable in the following ways: the first method is to apply pre-stretch on a dielectric elastomer with mechanical force [12, 14]; the second approach is to use a dielectric elastomer with interpenetrating networks $[22,23]$; the third technique is to swell a dielectric elastomer with a solvent [24]; the fourth means is to spray charges on an electrode-free elastomer [25]; the last 
one is to use an elastomer with a desirable stress-stretch curve [26], etc. In this study, the main attention is focused on the mechanical pre-stretch. In the static analysis, it is proved that the pre-stretch could eliminate or suppress the EMI, which significantly enhances the stability and contributes to the large actuation deformation $[14,15]$. Koh et al. [27] reported that the large deformation could also be achieved even the EMI is not eliminated, as long as the membrane could survive the 'snap-through deformation' without suffering the EB. In addition, by triggering the snap-through instability without causing EB, Keplinger et al demonstrated an expansion of area of $1692 \%$ for a dielectric membrane [28]. These findings offer a new method for the realization of large deformation.

Unfortunately, most of the research on the large actuation deformation of dielectric elastomers using pre-stretch were studied by the static analysis, without taking factors such as viscoelasticity, voltage actuation method and temperature into consideration $[14,15,27]$. As a time-dependent factor, viscoelasticity has a significant effect on the electromechanical performance of dielectric elastomers, which is proved by numerous research [29-36]. Besides, the voltage actuation method is obscure in the previous research using static analysis, while the deformation of the dielectric elastomer is various when it is actuated by different voltages. Furthermore, most of research about the dielectric elastomer is assumed to operate in an isothermal environment. It was reported that temperature also affects the electromechanical performances of dielectric elastomers to some extent [37-41]. Meanwhile, the dielectric constant of the elastomer is strongly related to the temperature, as it is 
proved by the experiments of Jean-Mistral C [42]. Thus, further study on the large actuation deformation for dissipative dielectric elastomers with the consideration of viscoelasticity, voltage actuation method and temperature is necessary to develop dielectric elastomers with excellent performance.

In this study, the mathematical model considering temperature and viscoelasticity is developed for dissipative dielectric elastomers. For the first time, the large actuation deformation of a dissipative dielectric elastomer using pre-stretch is studied for the two voltage actuation methods: the constant and ramping voltage actuation, respectively. First, at the reference temperature $T_{0}=300 \mathrm{~K}$, the deformation results are analyzed in detail for different combined conditions of pre-stretch and voltage. Then, the best deformation type and its corresponding combined conditions are obtained at three representative temperatures: $250 \mathrm{~K}, 300 \mathrm{~K}$ and $350 \mathrm{~K}$. Finally, under the best combined conditions, the effects of pre-stretch, voltage and temperature on the maximum actuation strain are investigated. This study strongly demonstrates that the optimal selection of conditions: pre-stretch, voltage and temperature is crucial to improve the performance of dielectric elastomers in the design of actuator.

\section{Working principle of dielectric elastomers}

(a) Reference state

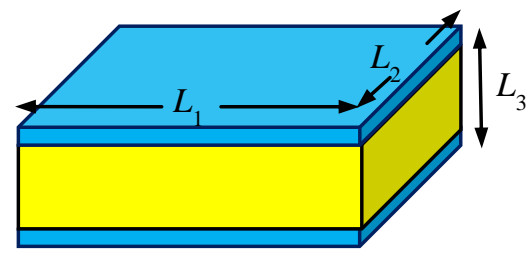

(b) Current state

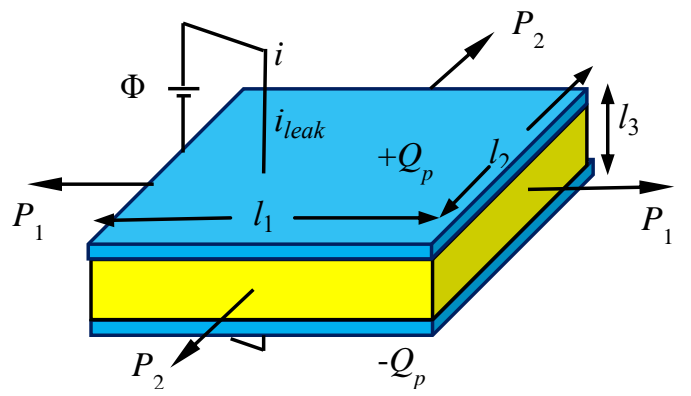


Figure 1. At the reference state, the dielectric elastomer is undeformed, and its dimensions are $L_{1}$, $L_{2}$ and $L_{3}$. At the current state, subject to mechanical forces $P_{1}$ and $P_{2}$ in its planar direction and a voltage $\Phi$ across its thickness direction, the membrane has dimensions $l_{1}, l_{2}$ and $l_{3}$.

Figure 1 illustrates the operation principle of a dielectric elastomer sandwiched between two electrodes with negligible electrical resistance and mechanical stiffness. At the reference state, the membrane is subjected to no force and voltage, and its dimension is $L_{1}, L_{2}$ and $L_{3}$. At the current state, the membrane is applied with forces $P_{1}$ and $P_{2}$, and the two electrodes are connected to a power source of voltage $\Phi$, with the dimensions changed to $l_{1}, l_{2}$ and $l_{3}$. During this process, the charges moving in the conducting wire, partially leak through the membrane and the rest accumulate on the electrodes to polarize the membrane [43]. The amount of polarizing charge on the electrodes is $Q_{p}$. The stretches of the elastomer in the three directions are defined by $\lambda_{1}=l_{1} / L_{1}, \lambda_{2}=l_{2} / L_{2}, \lambda_{3}=l_{3} / L_{3}$. The nominal stresses are defined by $s_{1}=P_{1} /\left(L_{2} L_{3}\right), s_{2}=P_{2} /\left(L_{1} L_{3}\right)$. The nominal electric field is $\widetilde{E}=\Phi / L_{3}$, and the nominal electric displacement is $\widetilde{D}=Q_{p} /\left(L_{1} L_{2}\right)$. When the elastomer experiences a large deformation, the change in the volume is much smaller than the change in the shape. Thus, the elastomer is assumed to be incompressible, namely $l_{1} l_{2} l_{3}=L_{1} L_{2} L_{3}$ and $\lambda_{1} \lambda_{2} \lambda_{3}=1$

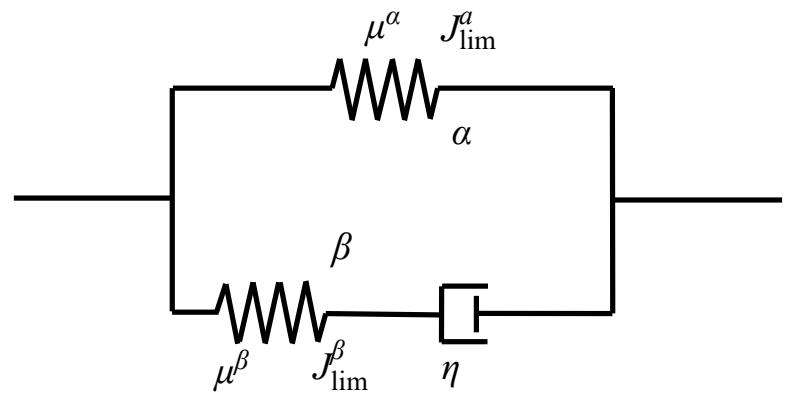

Figure 2. Viscoelasticity is modeled by two parallel units: the top unit consists of spring $\alpha$, and the other one consists of spring $\beta$ and a dashpot.

The viscoelasticity is represented by a rheological model of springs and dashpots 
with two parallel units [44-47]: one unit consists of a spring $\alpha$, and the other unit consists of spring $\beta$ and a dashpot (Fig. 2). The deformation of the elastomer is represented by in-plane stretches $\lambda_{1}$ and $\lambda_{2}$. In this rheological model, $\lambda_{1}$ and $\lambda_{2}$ are assumed to be the net stretches of both networks. For the spring $\alpha$, the state of deformation is characterized by stretches $\lambda_{1}$ and $\lambda_{2}$. While for the spring $\beta$, the state of deformation is characterized by stretches $\lambda_{1}^{e}$ and $\lambda_{2}^{e}$. Let $\xi_{1}$ and $\xi_{2}$ be the stretches in the dashpot, and the well-established multiplication rule [44, 46, 47] is adopted in this research, namely $\lambda_{1}=\lambda_{1}^{e} \xi_{1}, \lambda_{2}=\lambda_{2}^{e} \xi_{2}$.

\subsection{Constitutive model of dielectric elastomers}

The thermodynamic behavior of the dielectric elastomer is characterized by the Helmholtz free energy [48]. When the dimensions of the membrane change by $\delta l_{1}$, $\delta l_{2}$ and $\delta l_{3}$, the external forces do work $P_{1} \delta l_{1}+P_{2} \delta l_{2}$. As a small quantity of charge $\delta Q$ flows through the conducting wire, the voltage does work $\Phi \delta Q$. Thermodynamics requires that the increasing of the free energy $\delta F$ should not exceed the sum of work done by the force and the voltage, namely

$$
\delta F \leq P_{1} \delta l_{1}+P_{2} \delta l_{2}+\Phi \delta Q
$$

Dividing both sides of Eq. (1) by the volume of the membrane $L_{1} L_{2} L_{3}$, the thermodynamic inequality becomes

$$
\delta W \leq s_{1} \delta \lambda_{1}+s_{2} \delta \lambda_{2}+\tilde{E} \delta \tilde{D}
$$

where $W=F /\left(L_{1} L_{2} L_{3}\right)$ is the density of the Helmholtz free energy. For dissipative dielectric elastomers, the Helmholtz free energy is prescribed as a function related to six independent variables $\left(\lambda_{1}, \lambda_{2}, \xi_{1}, \xi_{2}, T, \widetilde{D}\right)$, with the viscoelasticity and temperature 
considered:

$$
W=W\left(\lambda_{1}, \lambda_{2}, \xi_{1}, \xi_{2}, T, \tilde{D}\right)
$$

The work process is assumed to be isothermal, so that the temperature $T$ remains the same in the process of actuation deformation. During the deformation, the state of a membrane can be characterized by $\lambda_{1}, \lambda_{2}, \widetilde{D}$, and the additional variables: $\xi_{1}, \xi_{2}$. From Eq. (2), it is known that the external loads do work through $\lambda_{1}, \lambda_{2}$ and $\widetilde{D}$. In contrast, $\xi_{1}$, and $\xi_{2}$ are the internal variables related to the dissipative process and have no relation to the external loads. When the independent variables $\left(\lambda_{1}, \lambda_{2}, \xi_{1}, \xi_{2}, \widetilde{D}\right)$ change by small amounts $\left(\delta \lambda_{1}, \delta \lambda_{2}, \delta \xi_{1}, \delta \xi_{2}, \delta \widetilde{D}\right)$, the free energy density changes by

$$
\delta W=\frac{\partial W}{\partial \lambda_{1}} \delta \lambda_{1}+\frac{\partial W}{\partial \lambda_{2}} \delta \lambda_{2}+\frac{\partial W}{\partial \tilde{D}} \delta \tilde{D}+\frac{\partial W}{\partial \xi_{1}} \delta \xi_{1}+\frac{\partial W}{\partial \xi_{2}} \delta \xi_{2}
$$

Inserting Eq. (4) into Eq. (2), the thermodynamic inequality can be expressed as

$$
\begin{aligned}
& \left(\frac{\partial W}{\partial \lambda_{1}}-s_{1}\right) \delta \lambda_{1}+\left(\frac{\partial W}{\partial \lambda_{2}}-s_{2}\right) \delta \lambda_{2}+\left(\frac{\partial W}{\partial \tilde{D}}-\tilde{E}\right) \delta \tilde{D} \\
& +\frac{\partial W}{\partial \xi_{1}} \delta \xi_{1}+\frac{\partial W}{\partial \xi_{2}} \delta \xi_{2} \leq 0
\end{aligned}
$$

This thermodynamic inequality should hold for any change in the independent variables $\left(\delta \lambda_{1}, \delta \lambda_{2}, \delta \xi_{1}, \delta \xi_{2}, \delta \widetilde{D}\right)$ as the time goes by. The membrane is assumed in mechanical and electrostatic equilibrium, so that the coefficients in front of $\delta \lambda_{1}, \delta \lambda_{2}$ and $\delta \widetilde{D}$ in Eq. (5) vanish, giving

$$
\begin{aligned}
& s_{1}=\frac{\partial W\left(\lambda_{1}, \lambda_{2}, \xi_{1}, \xi_{2}, T, \tilde{D}\right)}{\partial \lambda_{1}} \\
& s_{2}=\frac{\partial W\left(\lambda_{1}, \lambda_{2}, \xi_{1}, \xi_{2}, T, \tilde{D}\right)}{\partial \lambda_{2}}
\end{aligned}
$$




$$
\tilde{E}=\frac{\partial W\left(\lambda_{1}, \lambda_{2}, \xi_{1}, \xi_{2}, T, \tilde{D}\right)}{\partial \tilde{D}}
$$

Eqs. (6) - (8) constitute the equations of the state and provide the required forces and voltage to equilibrate with the elastomer at the state $\left(\lambda_{1}, \lambda_{2}, \xi_{1}, \xi_{2}, T, \widetilde{D}\right)$, once the specific free energy function $W\left(\lambda_{1}, \lambda_{2}, \xi_{1}, \xi_{2}, T, \widetilde{D)}\right.$ is prescribed.

The free energy model of the thermodynamic system can be expressed as [49, 50]

$$
\begin{aligned}
W\left(\lambda_{1}, \lambda_{2}, \xi_{1}, \xi_{2}, T, \tilde{D}\right)= & \frac{T}{T_{0}} W_{\text {stretch }}\left(\lambda_{1}, \lambda_{2}, \xi_{1}, \xi_{2}\right)+\rho_{0} c_{0} \\
& {\left[T-T_{0}-T \ln \left(\frac{T}{T_{0}}\right)\right]+\frac{\tilde{D}^{2}}{2 \varepsilon(T)} \lambda_{1}^{-2} \lambda_{2}^{-2} }
\end{aligned}
$$

The first term in the right-hand side of the equation is the thermo-elastic energy, where $W_{\text {stretch }}\left(\lambda_{1}, \lambda_{2}, \xi_{1}, \xi_{2}\right)$ is the isothermal free energy density due to stretches at the reference temperature $T_{0}[51,52]$. The second term is the purely thermal contribution, where $\rho_{0}$ and $c_{0}$ are the mass density and the specific heat capacity, respectively [52]. The third term represents the electrostatic energy of the elastomer, where $\varepsilon(T)=\varepsilon_{0} \varepsilon_{r}(T)$ is the dielectric constant of the membrane. In this expression, $\varepsilon_{0}=8.85 \times 10^{-12} \mathrm{~F} / \mathrm{m}$ denotes the vacuum dielectric constant, and $\varepsilon_{r}(T)$ represents the relative dielectric constant as a function of temperature [42]

$$
\varepsilon_{r}(T)=\varepsilon_{\infty}+\frac{N \mu^{2}}{3 \varepsilon_{\infty} k T}
$$

where $\varepsilon_{\infty}$ is the dielectric constant at high frequency, $N$ is the dipole density, $\mu$ is the dipole moment, and $k$ is the Boltzmann constant. According to the research of $\mathrm{C}$ Jean-Mistral [42], the expression of $\varepsilon_{r}(T)=2.26+690 / T$ is adopted to describe the temperature dependence of the dielectric constant. 
Considering the stress-stiffening effect of dissipative dielectric elastomers, both of the springs in Fig. 2 are represented by using the Gent model [53]. The free energy function due to the stretch is the sum of the contribution from the two springs [54]:

$$
\begin{aligned}
W_{\text {stretch }} & =-\frac{\mu^{\alpha} J_{\lim }^{\alpha}}{2} \log \left(1-\frac{\lambda_{1}^{2}+\lambda_{2}^{2}+\lambda_{1}^{-2} \lambda_{2}^{-2}-3}{J_{\lim }^{\alpha}}\right) \\
& -\frac{\mu^{\beta} J_{\lim }^{\beta}}{2} \log \left(1-\frac{\lambda_{1}^{2} \xi_{1}^{-2}+\lambda_{2}^{2} \xi_{2}^{-2}+\lambda_{1}^{-2} \lambda_{2}^{-2} \xi_{1}^{2} \xi_{2}^{2}-3}{J_{\lim }^{\beta}}\right)
\end{aligned}
$$

The first term in the right-hand side of the Gent model represents the elastic energy of the top spring, and the second term denotes the elastic energy of the bottom spring. $\mu^{\alpha}$ and $\mu^{\beta}$ are shear moduli of the two springs. $J_{\lim }^{\alpha}$ and $J_{\lim }^{\beta}$ are the constants related to the limiting stretches of the two springs.

Combining Eqs. (6)- (11), we obtain

$$
\begin{aligned}
s_{1}= & \frac{\partial W\left(\lambda_{1}, \lambda_{2}, \xi_{1}, \xi_{2}, T, \tilde{D}\right)}{\partial \lambda_{1}} \\
= & \frac{T}{T_{0}} \frac{\mu^{\alpha}\left(\lambda_{1}-\lambda_{1}^{-3} \lambda_{2}^{-2}\right)}{1-\left(\lambda_{1}^{2}+\lambda_{2}^{2}+\lambda_{1}^{-2} \lambda_{2}^{-2}-3\right) / J_{\lim }^{\alpha}} \\
& +\frac{T}{T_{0}} \frac{\mu^{\beta}\left(\lambda_{1} \xi_{1}^{-2}-\xi_{1}^{2} \xi_{2}^{2} \lambda_{1}^{-3} \lambda_{2}^{-2}\right)}{1-\left(\lambda_{1}^{2} \xi_{1}^{-2}+\lambda_{2}^{2} \xi_{2}^{-2}+\xi_{1}^{2} \xi_{2}^{2} \lambda_{1}^{-2} \lambda_{2}^{-2}-3\right) / J_{\lim }^{\beta}} \\
& -\frac{\tilde{D}^{2}}{\varepsilon_{0} \varepsilon_{r}(T)} \lambda_{1}^{-3} \lambda_{2}^{-2} \\
s_{2}= & \frac{\partial W\left(\lambda_{1}, \lambda_{2}, \xi_{1}, \xi_{2}, T, \tilde{D}\right)}{\partial \lambda_{2}} \\
= & \frac{T}{T_{0}} \frac{\mu^{\alpha}\left(\lambda_{2}-\lambda_{1}^{-2} \lambda_{2}^{-3}\right)}{1-\left(\lambda_{1}^{2}+\lambda_{2}^{2}+\lambda_{1}^{-2} \lambda_{2}^{-2}-3\right) / J_{\lim }^{\alpha}} \\
& +\frac{T}{T_{0}} \frac{\mu^{\beta}\left(\lambda_{2} \xi_{1}^{-2}-\xi_{1}^{2} \xi_{2}^{2} \lambda_{1}^{-2} \lambda_{2}^{-3}\right)}{1-\left(\lambda_{1}^{2} \xi_{1}^{-2}+\lambda_{2}^{2} \xi_{2}^{-2}+\xi_{1}^{2} \xi_{2}^{2} \lambda_{1}^{-2} \lambda_{2}^{-2}-3\right) / J_{\lim }^{\beta}} \\
& -\frac{\tilde{D}^{2}}{\varepsilon_{0} \varepsilon_{r}(T)} \lambda_{1}^{-2} \lambda_{2}^{-3}
\end{aligned}
$$




$$
\tilde{E}=\frac{\partial W\left(\lambda_{1}, \lambda_{2}, \xi_{1}, \xi_{2}, T, \tilde{D}\right)}{\partial \tilde{D}}=\frac{\tilde{D}}{\varepsilon_{0} \varepsilon_{r}(T)} \lambda_{1}^{-2} \lambda_{2}^{-2}
$$

Eqs. (12)- (14) constitute the thermodynamic equations of state of a dissipative dielectric elastomer, with viscoelasticity and temperature considered.

Once the membrane is in mechanical and electrostatic equilibrium, Eq. (5) becomes

$$
\frac{\partial W}{\partial \xi_{1}} \delta \xi_{1}+\frac{\partial W}{\partial \xi_{2}} \delta \xi_{2} \leq 0
$$

This thermodynamic inequality could be satisfied when a suitable relation between $\left(\delta \xi_{1}, \delta \xi_{2}\right)$ and $\left(\partial W / \partial \xi_{1}, \partial W / \partial \xi_{2}\right)$ is provided. According to the literature [55], $d \xi_{1} / d t=-\eta^{-1} \partial W / \partial \xi_{1}, d \xi_{2} / d t=-\eta^{-1} \partial W / \partial \xi_{2}$ are prescribed to meet the thermodynamic inequality. Inserting Eqs. (9) and (11) into the previous two expressions, we obtain that

$$
\begin{aligned}
\frac{d \xi_{1}}{d t} & =\frac{T}{T_{0}} \frac{\mu^{\beta}}{\eta} \frac{\lambda_{1}^{2} \xi_{1}^{-3}-\lambda_{1}^{-2} \lambda_{2}^{-2} \xi_{1} \xi_{2}^{2}}{1-\left(\lambda_{1}^{2} \xi_{1}^{-2}+\lambda_{2}^{2} \xi_{2}^{-2}+\lambda_{1}^{-2} \lambda_{2}^{-2} \xi_{1}^{2} \xi_{2}^{2}-3\right) / J_{\lim }^{\beta}} \\
& =\frac{T}{T_{0}} \frac{1}{\tau} \frac{\lambda_{1}^{2} \xi_{1}^{-3}-\lambda_{1}^{-2} \lambda_{2}^{-2} \xi_{1} \xi_{2}^{2}}{1-\left(\lambda_{1}^{2} \xi_{1}^{-2}+\lambda_{2}^{2} \xi_{2}^{-2}+\lambda_{1}^{-2} \lambda_{2}^{-2} \xi_{1}^{2} \xi_{2}^{2}-3\right) / J_{\lim }^{\beta}} \\
\frac{d \xi_{2}}{d t} & =\frac{T}{T_{0}} \frac{\mu^{\beta}}{\eta} \frac{\lambda_{2}^{2} \xi_{2}^{-3}-\lambda_{1}^{-2} \lambda_{2}^{-2} \xi_{1}^{2} \xi_{2}}{1-\left(\lambda_{1}^{2} \xi_{1}^{-2}+\lambda_{2}^{2} \xi_{2}^{-2}+\lambda_{1}^{-2} \lambda_{2}^{-2} \xi_{1}^{2} \xi_{2}^{2}-3\right) / J_{\lim }^{\beta}} \\
& =\frac{T}{T_{0}} \frac{1}{\tau} \frac{\lambda_{2}^{2} \xi_{2}^{-3}-\lambda_{1}^{-2} \lambda_{2}^{-2} \xi_{1}^{2} \xi_{2}}{1-\left(\lambda_{1}^{2} \xi_{1}^{-2}+\lambda_{2}^{2} \xi_{2}^{-2}+\lambda_{1}^{-2} \lambda_{2}^{-2} \xi_{1}^{2} \xi_{2}^{2}-3\right) / J_{\lim }^{\beta}}
\end{aligned}
$$

where $\tau$ is the viscoelastic relaxation time which is defined by the viscosity of the dashpot $\eta$ and the moduli of the bottom spring $\beta$ [54]:

$$
\tau=\frac{\eta}{\mu^{\beta}}
$$

In the analysis, the membrane is assumed to be subjected to a homogeneous and equal-biaxial force, making $\lambda_{1}=\lambda_{2}=\lambda, \xi_{1}=\xi_{2}=\xi$ and $s_{1}=s_{2}=s$. Thus, Eqs. (12)- (14) are 
written as follows:

$$
\begin{gathered}
s=\frac{T}{T_{0}} \frac{\mu^{\alpha}\left(\lambda-\lambda^{-5}\right)}{1-\left(2 \lambda^{2}+\lambda^{-4}-3\right) / J_{\lim }^{\alpha}} \\
+\frac{T}{T_{0}} \frac{\mu^{\beta}\left(\lambda \xi^{-2}-\xi^{4} \lambda^{-5}\right)}{1-\left(2 \lambda^{2} \xi^{-2}+\xi^{4} \lambda^{-4}-3\right) / J_{\lim }^{\beta}} \\
-\frac{\tilde{D}^{2}}{\varepsilon_{0} \varepsilon_{r}(T)} \lambda^{-5} \\
\tilde{E}=\frac{\tilde{D}}{\varepsilon_{0} \varepsilon_{r}(T)} \lambda^{-4}
\end{gathered}
$$

The rate of the stretch due to dashpot in Eqs. (16) and (17) can be expressed as

$$
\frac{d \xi}{d t}=\frac{T}{T_{0}} \frac{1}{\tau} \frac{\lambda^{2} \xi^{-3}-\lambda^{-4} \xi^{3}}{1-\left(2 \lambda^{2} \xi^{-2}+\lambda^{-4} \xi^{4}-3\right) / J_{\lim }^{\beta}}
$$

Combining Eqs. (19) and (20), we obtain

$$
\begin{aligned}
s+\varepsilon_{0} \varepsilon_{r}(T) \mathcal{E}^{2} \lambda^{3}= & \frac{T}{T_{0}} \frac{\mu^{\alpha}\left(\lambda-\lambda^{-5}\right)}{1-\left(2 \lambda^{2}+\lambda^{-4}-3\right) / J_{\lim }^{\alpha}} \\
& +\frac{T}{T_{0}} \frac{\mu^{\beta}\left(\lambda \xi^{-2}-\lambda^{-5} \xi^{4}\right)}{1-\left(2 \lambda^{2} \xi^{-2}+\lambda^{-4} \xi^{4}-3\right) / J_{\lim }^{\beta}}
\end{aligned}
$$

\subsection{Viscoelastic relaxation}

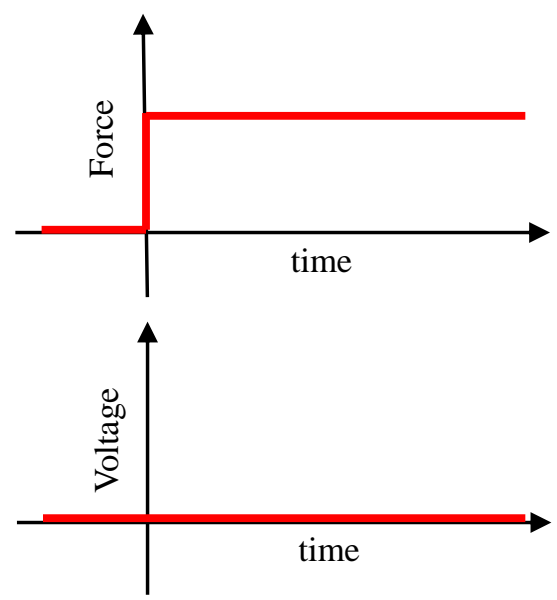

(a)

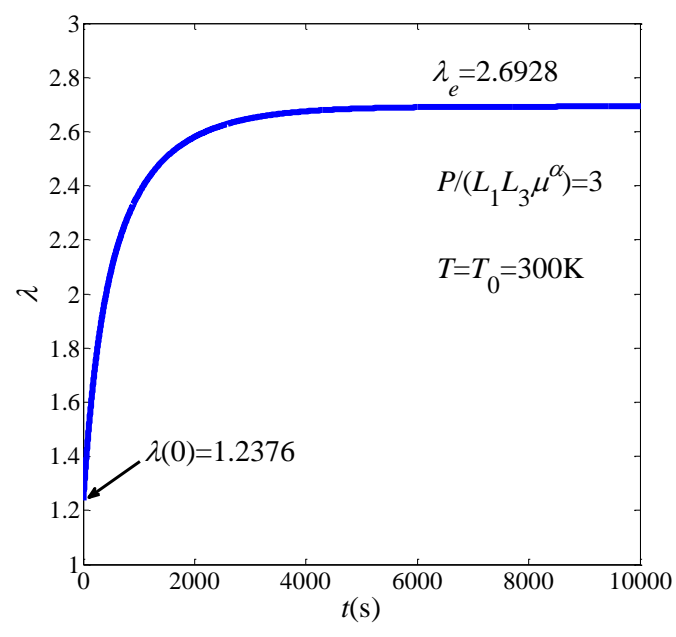

(b) 


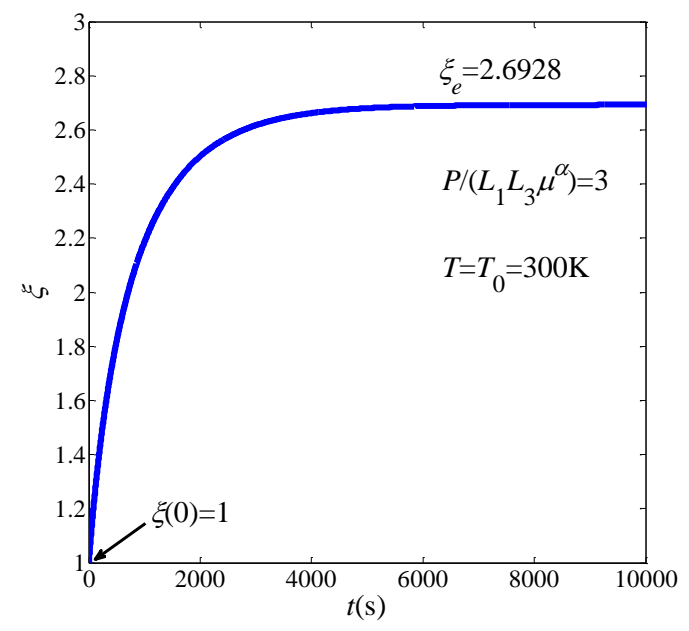

(c)

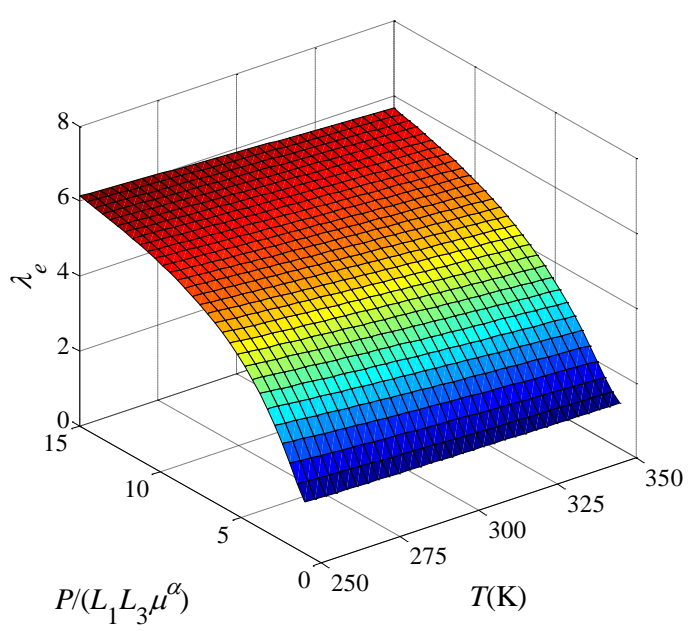

(d)

Figure 3. Under a constant mechanical load without voltage (a), the stretch of the membrane $\lambda$ (b) and the stretch due to the dashpot $\xi$ (c) creep in time. (d) The relationship between the equilibrium stretch $\lambda_{e}$, the force $P /\left(L_{1} L_{3} \mu^{\alpha}\right)$ and the temperature $T$.

To study the viscoelastic relaxation independently, a creep experiment at the reference temperature $T_{0}=300 \mathrm{~K}$ is modeled (Fig. 3). At time $t=0$, a force $P /\left(L_{1} L_{3} \mu^{\alpha}\right)=3$ is applied in the absence of voltage, and hold constant when the membrane creeps in time. The stretch of the dashpot $\xi$ does not move instantaneously, so that its initial value is $\xi(0)=1$. The initial stretch $\lambda(0)$ is solved from the nonlinear algebraic Eq. (22), by setting $\xi(0)=1, s=s(0), \widetilde{E}=0$ and $T=T_{0}=300 \mathrm{~K}$. With Eqs. (21) and (22), the evolutions of stretch $\lambda$ (Fig. 3(b)) and the stretch due to the dashpot $\xi$ (Fig. 3(c)) are obtained. In the simulations, a common set of parameters based on the existing experimental data of the VHB 4905/4910 elastomer is used: $\mu^{\alpha}=18 \mathrm{kPa}, \mu^{\beta}=42 \mathrm{kPa}, J_{\lim }^{\alpha}=110, J_{\lim }^{\beta}=55, \tau=400 \mathrm{~s}[43,54,56]$. As shown in Figs. 3(b) and (c), the force induces a small stretch $\lambda(0)=1.2367$ at the beginning state, and the load is carried by both spring $\alpha$ and spring $\beta$. Both the stretch $\lambda$ and the stretch due to the dashpot $\xi$ creep during the process of viscoelastic relaxation, and reach the equilibrium state after a period of time, with 
$\lambda_{e} \approx \xi_{e} \approx 2.6928$. After the equilibrium, the load is carried by the spring $\alpha$ alone. In addition, a series of creep experiments are modeled to study the influence of force and temperature on the viscoelastic relaxation. A sequence of forces varying from $P /\left(L_{1} L_{3} \mu^{\alpha}\right)=1$ to 15 is prescribed at different temperatures ranging from 250 to 300

$\mathrm{K}$. The range of temperature is selected based on Jean-Mistral's experiments [42]. For a specific condition of force and temperature, the equilibrium stretch $\lambda_{e}$ is recorded when the membrane reaches the equilibrium state as shown in Fig. 3(d). The equilibrium stretch $\lambda_{e}$ increases with the increase of the external force, but slightly decreases with the increase of temperature. If a large force is applied on the membrane at a low temperature, the equilibrium stretch $\lambda_{e}$ can be very large, which may result in a failure model known as rupture by stress [37].

\section{Deformation under voltage actuation}

In order to achieve a large actuation deformation for dissipative dielectric elastomers, two actuation methods: constant voltage and ramping voltage actuation are separately studied in this section. As all of the three factors: temperature, voltage and pre-stretch have effects on the deformation results of a dielectric elastomer, one of the three factors should be fixed in order to investigate the rest two factors. Thus, at the reference temperature $T_{0}=300 \mathrm{~K}$, the deformation results of the membrane under different combined conditions of voltage and pre-stretch are analyzed at first. Then, the best deformation type and its relation to the loading conditions: voltage and pre-stretch are obtained for the three representative temperatures: $250 \mathrm{~K}, 300 \mathrm{~K}$ and 
$350 \mathrm{~K}$. Finally, in the best combined conditions area, the effects of voltage, pre-stretch and temperature on the maximum actuation strain are discussed in detail.

In this section, equal-biaxial pre-stretch is applied before time $t=0$ by stretching a membrane to a certain level $\lambda_{p}$. Enough time is provided for the viscoelastic relaxation to be fully accomplished, so that the membrane could gain an equilibrium state at time $t=0$, with $\lambda(0)=\xi(0)=\lambda_{p}$. Then, the pre-stress $s_{p}=P /\left(L_{1} L_{3}\right)$ is fixed and the voltage is applied at time $t=0$. The corresponding pre-stress $s_{p}$ could be calculated by Eq. (22), setting $\lambda(0)=\xi(0)=\lambda_{p}$ and $\widetilde{E}=0$. If the pre-stretch is prescribed as $\lambda_{p}=1$, the corresponding stress is $s_{p}=0$, which implies that no pre-stretch is applied. Once a voltage is prescribed at the time $t=0$, the evolution of stretch $\lambda$ could be obtained by solving Eqs. (21) and (22). Using the equation $E=\lambda^{2} \widetilde{E}$, the evolution of the true electric field $E$ could also be attained to judge the happening of EB. Here the EB strength $E_{E B}$ is assumed to be independent of deformation, and $E_{E B}=3 \times 10^{8} \mathrm{~V} / \mathrm{m}$ is adopted $[12,26,57,58]$. In order to investigate the large deformation of dielectric elastomers, the maximum actuation strain is defined as $\left(\lambda_{\max } / \lambda_{p}-1\right) \times 100 \%$, where $\lambda_{\max }$ is the maximum stretch without failure.

\subsection{Constant voltage actuation deformation}
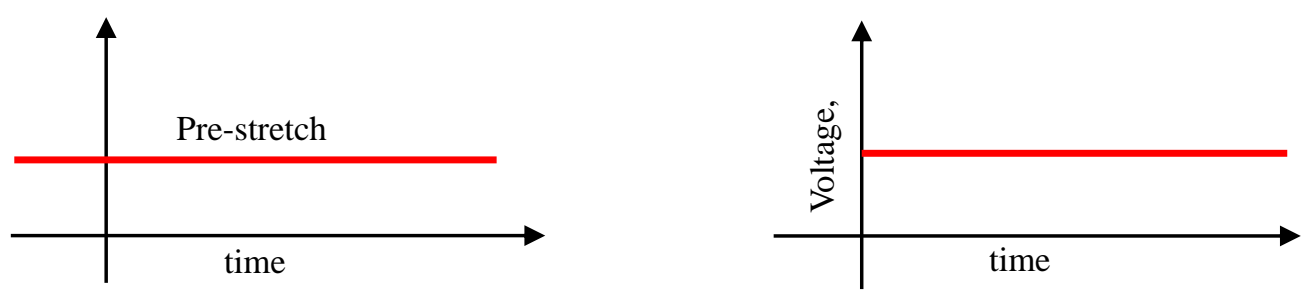

Figure 4. Loading program

Figure 4 shows the loading program: a constant voltage is applied on the 
membrane at the time $t=0$ after a pre-stretch $\lambda_{p}$. In the simulation, the constant voltage $\Phi$ is applied as a form of the nominal electric field $\widetilde{E}=\Phi / L_{3}$. At the reference temperature $T_{0}=300 \mathrm{~K}$, without loss of generality, five representative pre-stretch conditions: $\lambda_{p}=1,1.2,1.6,2.14$ and 4 are separately studied. The deformation results of dielectric elastomers under different combined conditions of constant voltage and pre-stretch are shown in Fig. 5. The specific pre-stretches are selected to present typical deformation results of the membrane applied voltages from small to large. The deformation of pre-stretch $\lambda_{p}=1$ is used to show the deformation results for no pre-stretch condition. The rest of four selected pre-stretches also typically illustrate the deformation results for the four pre-stretch ranges: $\lambda_{p}=1 \sim 1.44$, 1.45 2.11, 2.12 2.58 and 2.59 4.
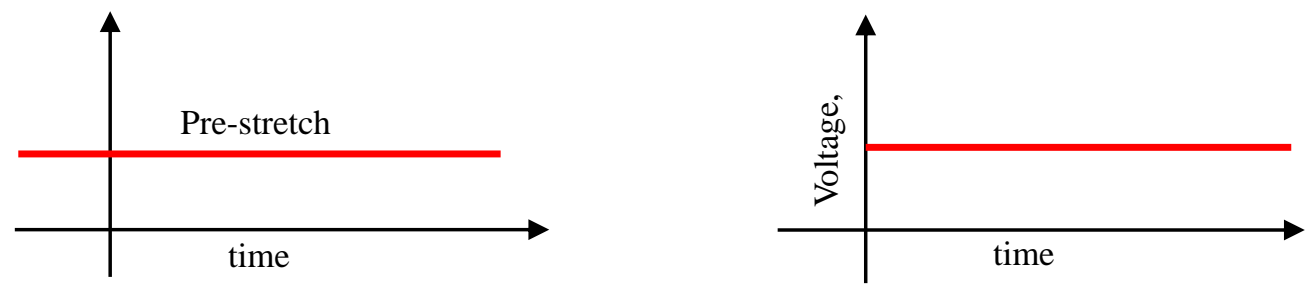

Figure 4. Loading program

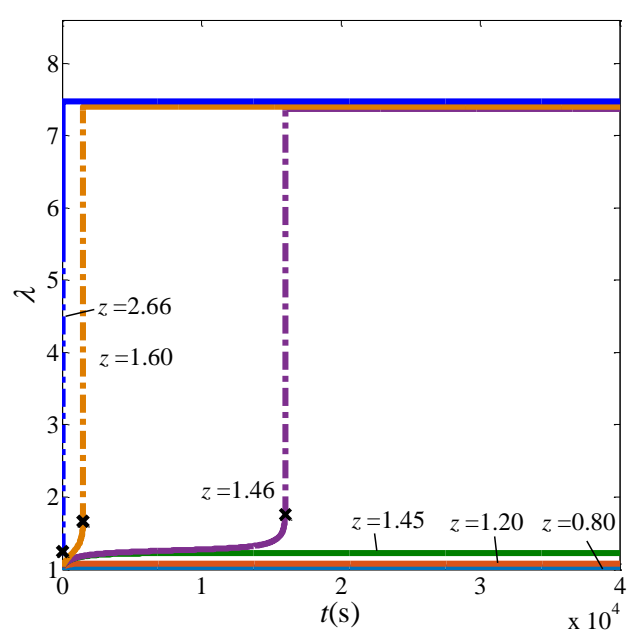

(a) $\lambda_{p}=1, T_{0}=300 \mathrm{~K}$

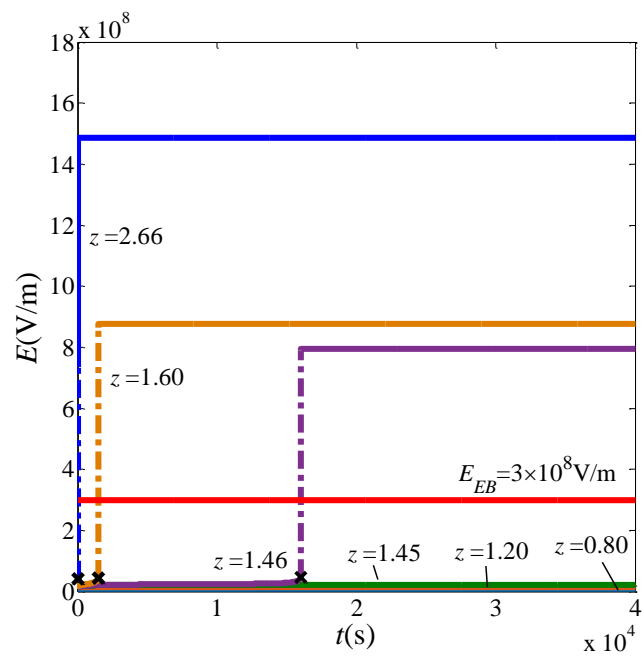

(b) $\lambda_{p}=1, T_{0}=300 \mathrm{~K}$ 


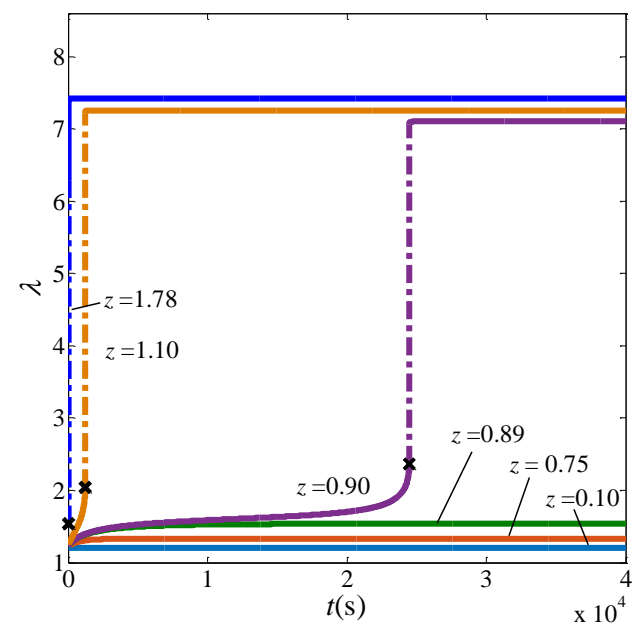

(c) $\lambda_{p}=1.2, T_{0}=300 \mathrm{~K}$

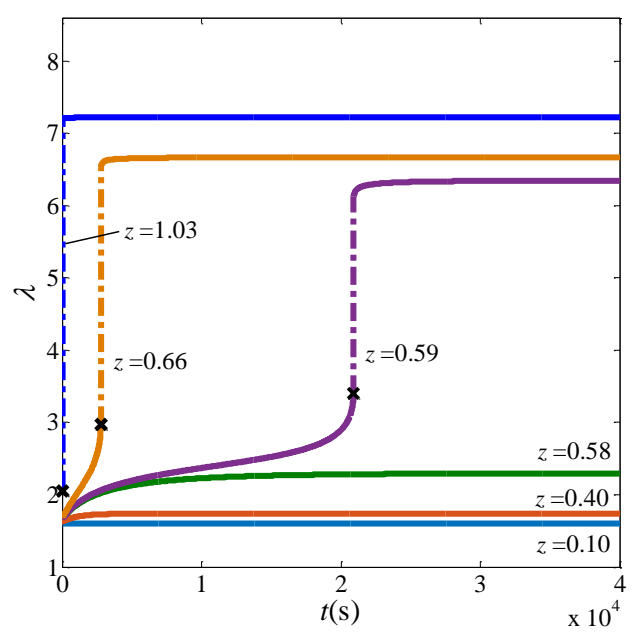

(e) $\lambda_{p}=1.6, T_{0}=300 \mathrm{~K}$

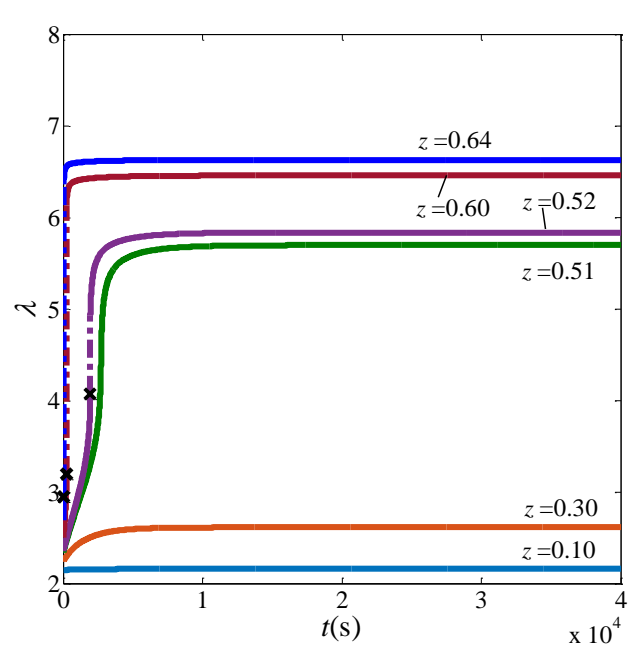

(g) $\lambda_{p}=2.14, T_{0}=300 \mathrm{~K}$

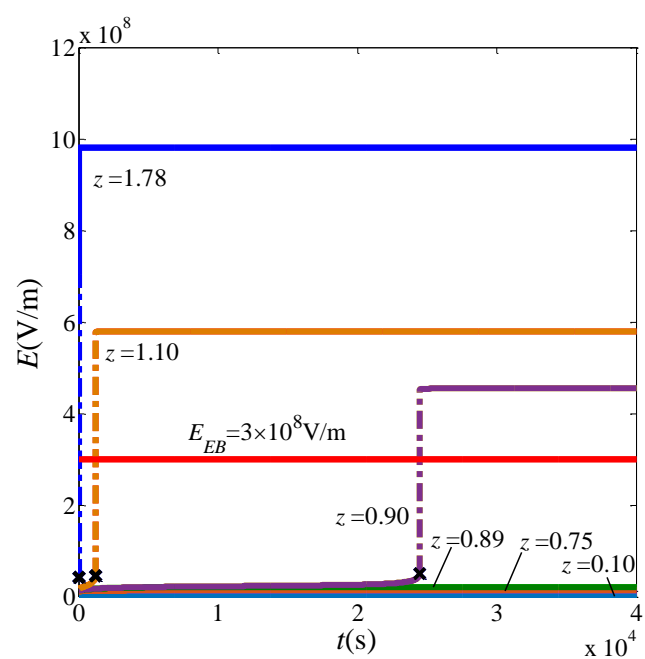

(d) $\lambda_{p}=1.2, T_{0}=300 \mathrm{~K}$

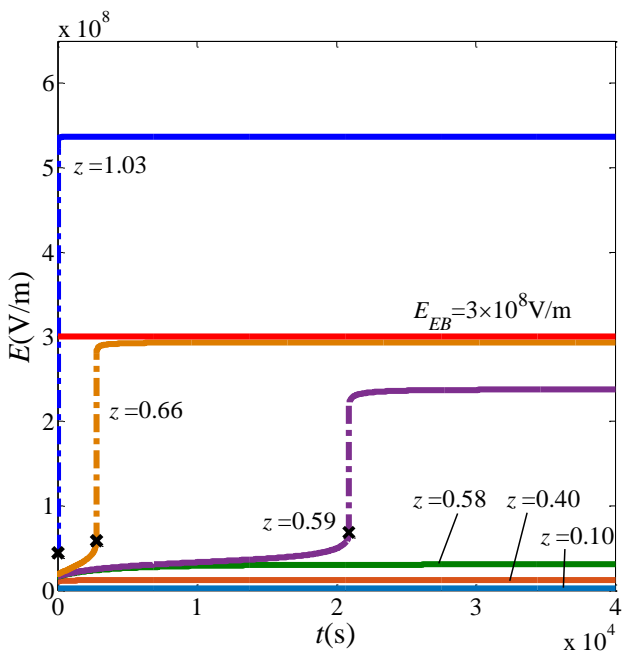

(f) $\lambda_{p}=1.6, T_{0}=300 \mathrm{~K}$

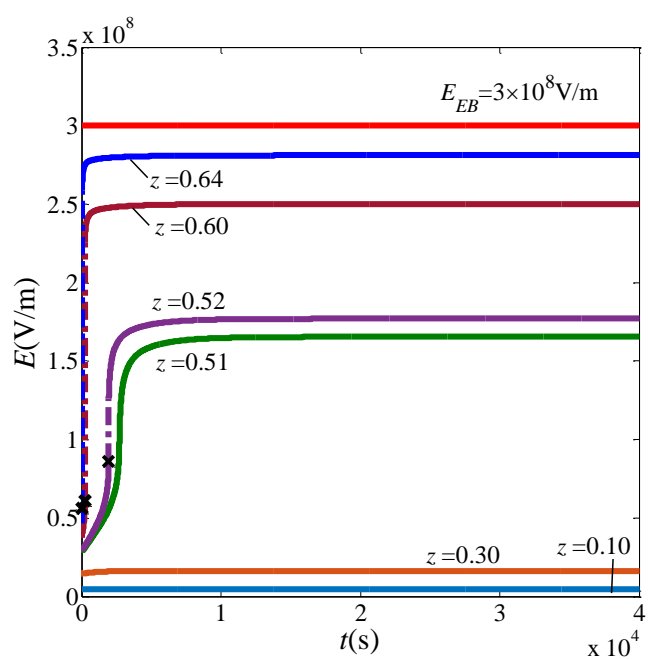

(h) $\lambda_{p}=2.14, T_{0}=300 \mathrm{~K}$ 


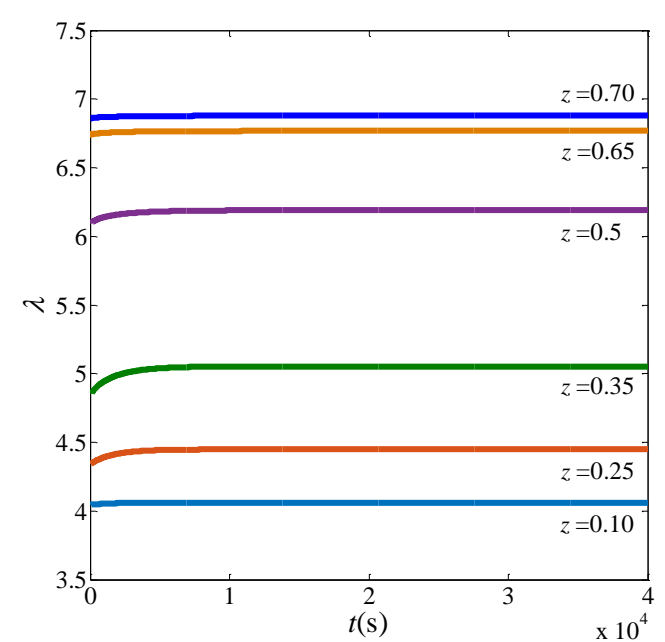

(i) $\lambda_{p}=4, T_{0}=300 \mathrm{~K}$

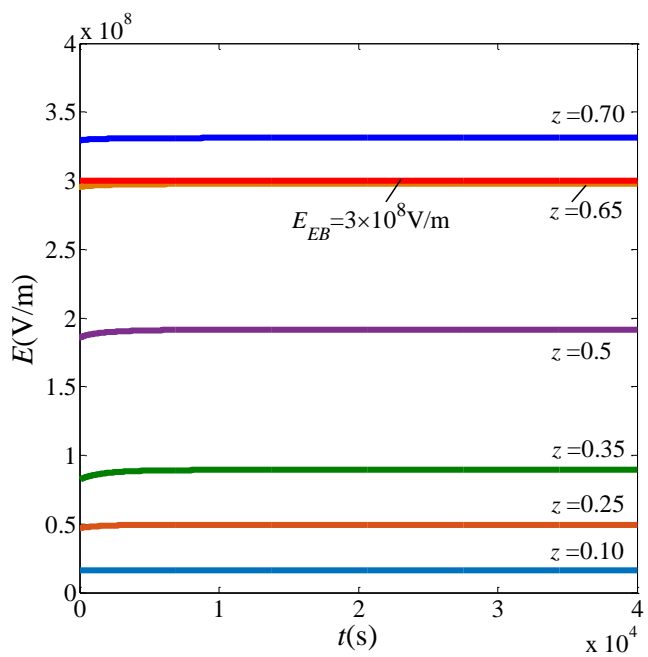

(j) $\lambda_{p}=4, T_{0}=300 \mathrm{~K}$

Figure 5. The evolution of stretch $\lambda$ ((a), (c), (e), (g), (i)) and the true electric field $E=\lambda^{2} \widetilde{E}$ ((b), (d), (f), (h), (j)) when the membrane is applied with different voltages after various pre-stretches. The applied nominal electric field $\widetilde{E}=z \times 10^{7} \mathrm{~V} / \mathrm{m}$. The crosses denote the EMI points, the vertical dashed lines represent the 'snap-through deformation', and the red horizontal line is the EB strength $E_{E B}$.

Under the condition of no pre-stretch $\left(\lambda_{p}=1\right)$, the membrane tends to have various deformation results when different constant voltages are applied (Figs. 5(a) and (b)). The membrane could gain an equilibrium state if $\widetilde{E}<1.46 \times 10^{7} \mathrm{~V} / \mathrm{m}$. When $1.46 \times 10^{7} \leq \widetilde{E} \leq 2.66 \times 10^{7} \mathrm{~V} / \mathrm{m}$, the membrane would suffer EMI when it reaches the critical stretch $\lambda_{c}$, which is denoted by the crosses in Figs. 5(a) and (b). Then, the stretch $\lambda$ rapidly jumps from a small stretch to a much larger one, with the true electric field $E$ increasing to a large value dramatically. This process is known as the 'snap-through deformation' $[59,60]$ and is represented by the vertical dashed line. However, all the membranes suffered EMI could not survive the 'snap-through deformation' and definitely fail as $\mathrm{EB}$, because the increasing true electric field $E$ surpasses the electrical breakdown strength $E_{E B}=3 \times 10^{8} \mathrm{~V} / \mathrm{m}$ during the 'snap-through deformation' process (the vertical dashed lines have cross with the red horizontal line which represents $E_{E B}$ in Fig. 5(b)). If $\widetilde{E}>2.66 \times 10^{7} \mathrm{~V} / \mathrm{m}$, the EMI would occur instantly as soon as the voltage is applied, and the membrane fails 
immediately. This is because the big electric field induced by a large voltage leads to a rapid decrease in the thickness direction, which results in EB. Similar deformation results could be found in the research of Zhao [55], in which a viscoelastic dielectric elastomer is prescribed to be applied with different constant voltages without pre-stretch. Under the condition of no pre-stretch, large deformation could be achieved within the voltage range: $1.46 \times 10^{7} \leq \widetilde{E} \leq 2.66 \times 10^{7} \mathrm{~V} / \mathrm{m}$, and the maximum actuation strain $\lambda_{\max }$ is determined by the critical stretch $\lambda_{c}$ when the EMI sets in. Within the voltage range, $\lambda_{c}$ increases with the decrease of voltage (Fig. 5(a)). Thus, under the conditions of no pre-stretch $\left(\lambda_{p}=1\right)$ and the reference temperature $T_{0}=300 \mathrm{~K}$, the maximum actuation strain could reach up to $75 \%\left(\widetilde{E}=1.46 \times 10^{7} \mathrm{~V} / \mathrm{m}, \lambda_{\max }=1.756\right)$.

The deformation results of the membrane applied with the small pre-stretch $\lambda_{p}=1.2$ (Fig. 5(c) and (d)) are similar to that of the no pre-stretch condition: the membrane could reach an equilibrium state if $\widetilde{E}<0.90 \times 10^{7} \mathrm{~V} / \mathrm{m}$; within the range of $0.9 \times 10^{7} \leq \widetilde{E} \leq 1.78 \times 10^{7} \mathrm{~V} / \mathrm{m}$, the membrane would experience EMI and 'snap-through deformation' after a period of time, and fails as EB in the end; As $\widetilde{E}>1.78 \times 10^{7} \mathrm{~V} / \mathrm{m}$, the membrane will fail as EMI instantaneously. The maximum actuation strain in the case of $\lambda_{p}=1.2$ could be up to $97 \%\left(\widetilde{E}=0.9 \times 10^{7} \mathrm{~V} / \mathrm{m}, \lambda_{\max }=2.366\right)$, which is larger than the one without pre-stretch $(75 \%)$.

The evolution of the stretch $\lambda$ and the true electric field $E$ are presented in Figs. 5(e) and (f), as the membrane is applied with different voltages after the pre-stretch $\lambda_{p}=1.6$. The membrane could gain an equilibrium state if $\widetilde{E}<0.59 \times 10^{7} \mathrm{~V} / \mathrm{m}$. When $0.59 \times 10^{7} \leq \widetilde{E} \leq 0.66 \times 10^{7} \mathrm{~V} / \mathrm{m}$, it will suffer EMI. Different from the case of a small 
pre-stretch $\left(\lambda_{p}=1.2\right)$ or without pre-stretch $\left(\lambda_{p}=1\right)$ conditions, the membrane could survive the 'snap-through deformation' after suffering EMI, as the true electric field $E$ remains below $E_{E B}=3 \times 10^{8} \mathrm{~V} / \mathrm{m}$ during the whole process of 'snap-through deformation'(Fig. 5(f)), without failing as EB. Instead, it could reach an equilibrium state with the stretch $\lambda_{\text {equ }}$, which is also the maximum stretch $\lambda_{\max }$. In contrast, within the range of $0.66 \times 10^{7}<\widetilde{E} \leq 1.03 \times 10^{7} \mathrm{~V} / \mathrm{m}$, the membrane will suffer EMI but could not survive the 'snap-through deformation', leading to EB finally. If $\widetilde{E}>1.03 \times 10^{7} \mathrm{~V} / \mathrm{m}$, the membrane will suffer EMI and fail as soon as the voltage is applied. Under this pre-stretch condition, the large deformation could be realized when $0.59 \times 10^{7} \leq \widetilde{E} \leq 0.66 \times 10^{7} \mathrm{~V} / \mathrm{m}$. The corresponding maximum actuation strain is determined by the equilibrium stretch $\lambda_{\text {equ }}$ after surviving the 'snap-through deformation'. As shown in Fig. 5(e), $\lambda_{\text {equ }}$ increases with the increase of the applied voltage. Thus, the maximum actuation strain could reach up to $316 \%$ $\left(\widetilde{E}=1.03 \times 10^{7} \mathrm{~V} / \mathrm{m}, \lambda_{\max }=6.669\right)$, which is more than 4 times larger than that in the case of no pre-stretch condition $(76 \%)$.

In the case of $\lambda_{p}=2.14$, the membrane could reach an equilibrium state if $\widetilde{E}<0.52 \times 10^{7} \mathrm{~V} / \mathrm{m}$ (Figs. $5(\mathrm{~g})$ and (h)). When $0.52 \times 10^{7} \leq \widetilde{E} \leq 0.64 \times 10^{7} \mathrm{~V} / \mathrm{m}$, it could survive the 'snap-through deformation' after suffering EMI. Finally, the membrane could gain an equilibrium stretch $\lambda_{\text {equ }}$, which increases as the increase of the applied voltage. If $\widetilde{E}>0.64 \times 10^{7} \mathrm{~V} / \mathrm{m}$, EMI will occur immediately, and the membrane will fail as soon as the voltage is applied. For the condition of $\lambda_{p}=2.14$, the maximum actuation strain could reach to $209 \%\left(\widetilde{E}=0.64 \times 10^{7} \mathrm{~V} / \mathrm{m}, \lambda_{\max }=6.627\right)$. 
As outlined in Figs. 5(i) and (j), under the pre-stretch condition $\lambda_{p}=4$, the membrane could reach an equilibrium state after a period of time as $\widetilde{E} \leq 0.65 \times 10^{7} \mathrm{~V} / \mathrm{m}$. However, it will fail as EB as soon as the voltage is applied if $\widetilde{E}>0.65 \times 10^{7} \mathrm{~V} / \mathrm{m}$. The reason is that the true electric field $E$ dramatically increases above $E_{E B}$ for the sharp decrease in the thickness direction. Unlike all the deformation results under various pre-stretch conditions mentioned above, the EMI and 'snap-through deformation' are eliminated by the large pre-stretch. The maximum stretch $\lambda_{\max }$ is the equilibrium stretch $\lambda_{\text {equ }}$ without failure, which increases with the increase of voltage within the relatively small range $\widetilde{E} \leq 0.65 \times 10^{7} \mathrm{~V} / \mathrm{m}$. When the pre-stretch $\lambda_{p}=4$, the maximum actuation strain could reach up to $69 \%\left(\widetilde{E}=0.65 \times 10^{7} \mathrm{~V} / \mathrm{m}, \lambda_{\max }=6.768\right)$, which is even smaller than the one without pre-stretch (76\%). This implies that the larger pre-stretch does not always ensure the increase of the maximum actuation strain. Thus, the optimal pre-stretch is crucial to improve the performance of dielectric elastomers as actuators.

Table 1. Deformation results for dielectric elastomers with various pre-stretches subjecting different constant voltages at the reference temperature $T_{0}=300 \mathrm{~K}$

\begin{tabular}{ccccc}
\hline \hline$\lambda_{p}$ & \multicolumn{2}{c}{ Deformation results with constant voltages from small to large } \\
\hline 1.2 & equilibrium & $\begin{array}{c}\text { EMI \& failure (maximum } \\
\text { actuation strain up to 75\%) }\end{array}$ & $\begin{array}{c}\text { EMI instantly \& } \\
\text { failure } \\
\text { EMI \& failure (maximum } \\
\text { EMI instantly \& } \\
\text { failure }\end{array}$ & \\
1.6 & equilibrium & $\begin{array}{c}\text { Ectuation strain up to 97\%) } \\
\text { EMI \& equilibrium } \\
\text { (maximum actuation strain } \\
\text { up to 316\%) } \\
\text { EMI \& equilibrium }\end{array}$ & EMI \& failure & $\begin{array}{c}\text { EMI instantly } \\
\text { \& failure }\end{array}$ \\
& $\begin{array}{c}\text { equilibrium } \\
\text { (maximum actuation strain } \\
\text { up to 209\%) }\end{array}$ & $\begin{array}{c}\text { EMI instantly \& } \\
\text { failure }\end{array}$ & \\
& $\begin{array}{c}\text { (maximum } \\
\text { actuation strain up } \\
\text { to 69\%) }\end{array}$ & EB instantly \& failure & & \\
\hline \hline
\end{tabular}

All the deformation results of dielectric elastomers under different constant 
voltages with the pre-stretches: $1,1.2,1.6,2.14$, and 4 are concluded in Tab. 1 . It is indicated that the deformation results of the membrane are dependent on the combined conditions of pre-stretch and voltage. Therefore, after substantial calculation, the relations between the deformation results and the combined conditions of pre-stretch and voltage are presented in Fig.6 for the three representative temperatures: $250 \mathrm{~K}, 300 \mathrm{~K}$ and $350 \mathrm{~K}$, respectively. Each point $\left(\lambda_{\mathrm{p}}, \widetilde{E}\right)$ on the diagram represents a combined condition of pre-stretch and voltage, and each region $\left(\mathrm{A}, \mathrm{B}_{1}\right.$, $\left.\mathrm{B}_{2}, \mathrm{C}, \mathrm{D}, \mathrm{E}\right)$ enclosed by the lines has the same deformation type. As depicted in Fig 6 , the layouts of the phase diagrams for the three temperatures are almost the same. Thus, the phase diagram of the reference temperature $T=300 \mathrm{~K}$ is firstly discussed in detail. Then, the phase diagrams of different temperature conditions are compared in the following analysis.

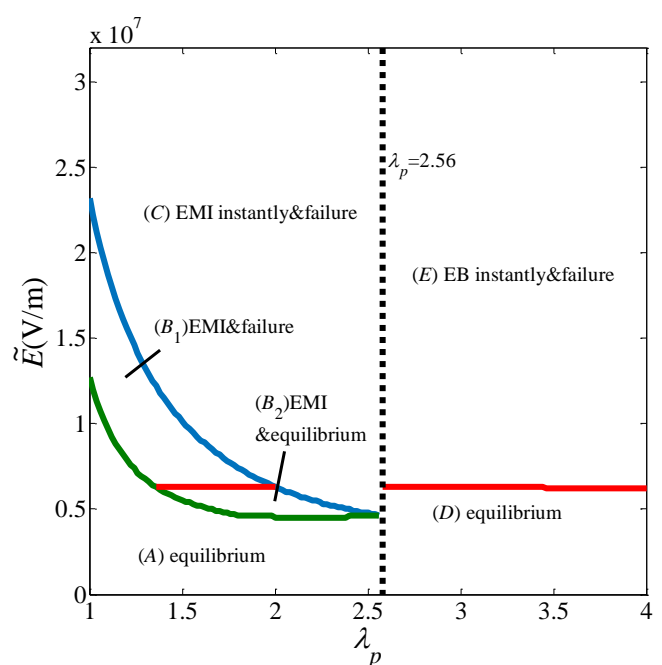

(a) $T=250 \mathrm{~K}$

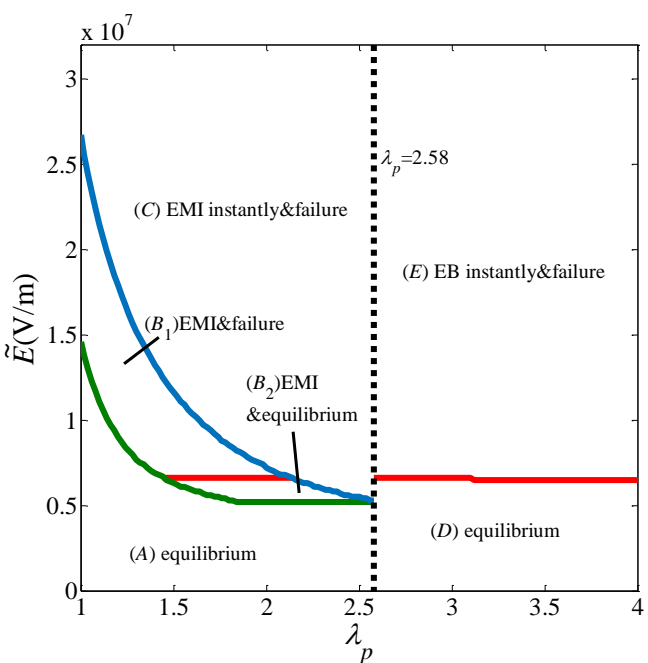

(b) $T=300 \mathrm{~K}$ 


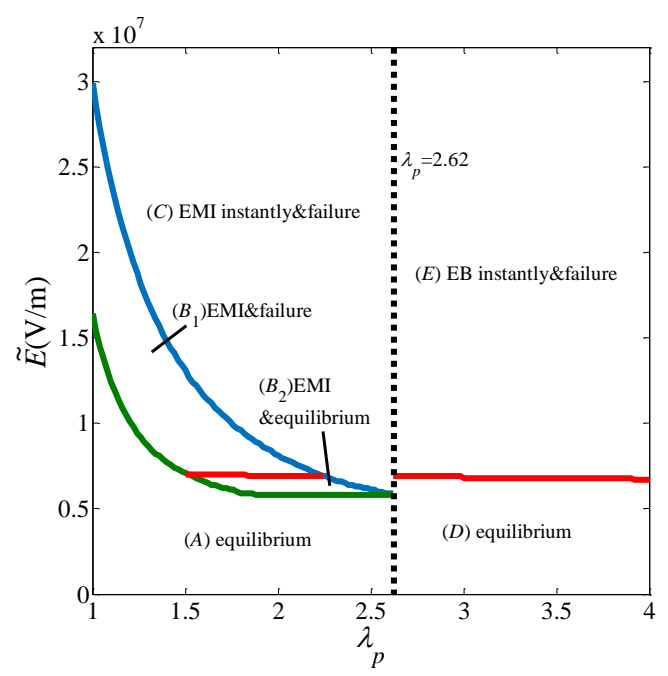

(c) $T=350 \mathrm{~K}$
Figure 6. Phase diagrams ( $T=250 \mathrm{~K}, T=300 \mathrm{~K}$, $T=350 \mathrm{~K})$ for the combined conditions of pre-stretch $\lambda_{p}$ and the nominal electric field $\widetilde{E}$ to the results of deformation A point $\left(\lambda_{p}, \widetilde{E}\right)$ on the diagrams denotes a specific combined condition of pre-stretch $\lambda_{p}$ and voltage. The vertical dashed lines indicate the minimum pre-stretch to eliminate the EMI and 'snap-through'

At the reference temperature $T=300 \mathrm{~K}$, the deformation results of the membrane shown in the Tab. 1 for pre-stretches: $\lambda_{p}=1.2,1.6,2.14$ and 4 under different constant voltages from small to large ones are the examples for the pre-stretch ranges: $\lambda_{p}=1 \sim 1.44,1.45 \sim 2.11,2.12 \sim 2.58,2.59 \sim 4$ in Fig. 6(b), respectively. This is the reason why the four pre-stretches are selected as the representative pre-stretches to be illustrated in Fig. 5. For instance, given a pre-stretch $\lambda_{p}$ within the range 1.45 2.11, there are four types of deformation results of the membrane when it is applied with constant voltages from small to large, that is equilibrium, EMI \& equilibrium, EMI \& failure and EMI instantly \& failure. These results are the same as that of pre-stretch $\lambda_{p}=1.6$ shown in Tab. 1 .

As shown in Fig. 6(b), at the reference temperature $T=300 \mathrm{~K}$, the EMI and 'snap-through deformation' are eliminated by pre-stretch $\lambda_{p}>2.58$. In the regions $(C)$ and $(E)$, the applied voltages are relatively large, and the membrane will fail instantly either by EMI $\left(\lambda_{p}<2.58\right)$ or by EB $\left(\lambda_{p} \geq 2.58\right)$. Thus, the combined conditions in these two regions should be avoided in the design of dielectric elastomers as actuators. As $\lambda_{p}<2.58$, the largest allowable nominal electric field $\widetilde{E}$ that makes the membrane 
exempt from the instant failure (the blue line in Fig. 6(b)) decreases with the increase of the pre-stretch $\lambda_{p}$. Thus, once the pre-stretch is increased, the applied voltage should be decreased correspondingly. Likewise, the maximum nominal electric field $\widetilde{E}$ for the membrane to reach an equilibrium state without suffering EMI (the green line) also decreases with the increasing pre-stretch. The dividing line between the regions $\left(B_{1}\right)$ and $\left(B_{2}\right)$, which decides whether the membrane could survive the 'snap-through deformation' and reach an equilibrium state or not, is around $\widetilde{E}=0.66 \times 10^{7} \mathrm{M} / \mathrm{v}$. Similarly, the dividing line between the regions $(D)$ and $(E)$ is about $\widetilde{E}=0.65 \times 10^{7} \mathrm{M} / \mathrm{v}$.

By comparing the magnitudes of maximum actuation strain under different combined conditions of pre-stretch and voltage shown in Tab. 1, it is observed that the best combined conditions to obtain the largest maximum actuation strain are located in the region $\left(B_{2}\right)$. As outlined in Tab. 1 and Fig. 6, prescribed with the combined conditions in this region, the membrane could reach an equilibrium state after suffering EMI and surviving the 'snap-through deformation'. Under this condition, the maximum actuation strain $316 \%$ is attainable $\left(\lambda_{p}=1.6, \widetilde{E}=1.03 \times 10^{7} \mathrm{~V} / \mathrm{m}\right.$, $\lambda_{\max }=6.669, T_{0}=300 \mathrm{~K}$ ), which is 4 times larger than that of no pre-stretch condition $(75 \%)$.

It is indicted from Fig. 6 that all the dividing lines increase with the increase of temperature from $250 \mathrm{~K}$ to $350 \mathrm{~K}$. For instance, under the no pre-stretch condition $\lambda_{p}=1$, the dividing nominal electric field between regions $(A)$ and $\left(B_{1}\right)$ is increased from $1.27 \times 10^{7} \mathrm{~V} / \mathrm{m}(250 \mathrm{~K})$ to $1.64 \times 10^{7} \mathrm{~V} / \mathrm{m}(350 \mathrm{~K})$. In addition, the minimum 
pre-stretch that eliminates EMI and 'snap-through deformation' also experiences a slight increase from $\lambda_{p}=2.56$ to $\lambda_{p}=2.62$ as the temperature increases from $250 \mathrm{~K}$ to $300 \mathrm{~K}$.

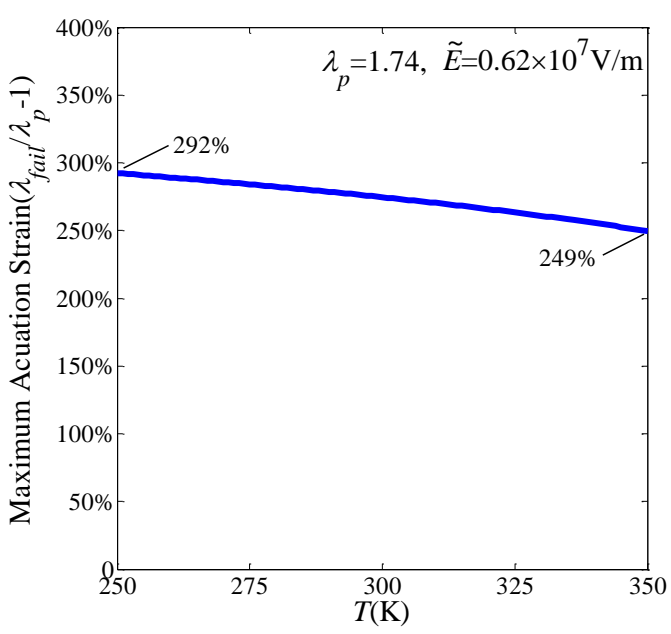

(a)

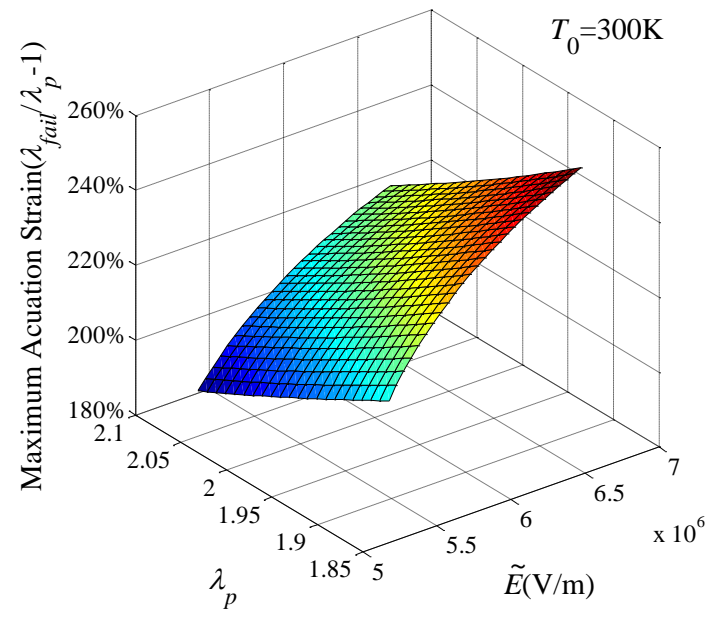

(b)

Figure 7. (a) The maximum actuation strain in relation to the temperature as $\lambda_{p}=1.74$ and $\widetilde{E}=0.62 \times 10^{7} \mathrm{~V} / \mathrm{m}$. (b) The maximum actuation strain in relation to pre-stretch $\lambda_{p}$ and nominal electric field $\widetilde{E}$ at the reference temperature $T_{0}=300 \mathrm{~K}$.

As the region $\left(B_{2}\right)$ in Fig. 6 has the largest maximum actuation strain, it is necessary to analyze the effects of factors: pre-stretch, constant voltage and temperature on the maximum actuation strain in this region. Firstly, $\lambda_{p}=1.74$ and $\widetilde{E}=1.03 \times 10^{7} \mathrm{~V} / \mathrm{m}$ are chosen to ensure that the combined condition $\left(\lambda_{p}, \widetilde{E}\right)$ is located in the region $\left(B_{2}\right)$ for all the temperature conditions ranging from $250 \mathrm{~K}$ to $350 \mathrm{~K}$. This condition makes sure that, the dielectric elastomer could survive the 'snap-through deformation' and obtains a large actuation deformation within the whole temperature range $250 \sim 350 \mathrm{~K}$. As shown in Fig. 7(a), when the temperature increases from $250 \mathrm{~K}$ to $350 \mathrm{~K}$, the maximum actuation strain decreases from $292 \%$ to $249 \%$, indicating that the maximum actuation strain decreases with the increase of the temperature. In addition, at the reference temperature $T_{0}=300 \mathrm{~K}$, the maximum 
actuation strain is also plotted in Fig. 7(b) with the conditions: $1.87 \leq \lambda_{p} \leq 2.08$, $0.53 \times 10^{7} \leq \widetilde{E} \leq 0.66 \times 10^{7} \mathrm{~V} / \mathrm{m}$. These pre-stretch and nominal electric field ranges are also specially selected to ensure the coordinate $\left(\lambda_{p}, \widetilde{E}\right)$ always located within the region $\left(B_{2}\right)$ (Fig. 6(b)) at the reference temperature $T_{0}=300 \mathrm{~K}$, which ensures the realization of large actuation deformation. As indicated from Fig. 7(b), a large maximum actuation strain up to $257 \%$ is achieved using a relatively large nominal electric field $\widetilde{E}=0.66 \times 10^{7} \mathrm{~V} / \mathrm{m}$ with a small pre-stretch $\lambda_{p}=1.87$. In conclusion, under the best combined conditions of pre-stretch and voltage, the maximum actuation strain increases with the increase of the constant voltage, but decreases with the increase of pre-stretch and temperature.

\subsection{Ramping voltage actuation deformation}

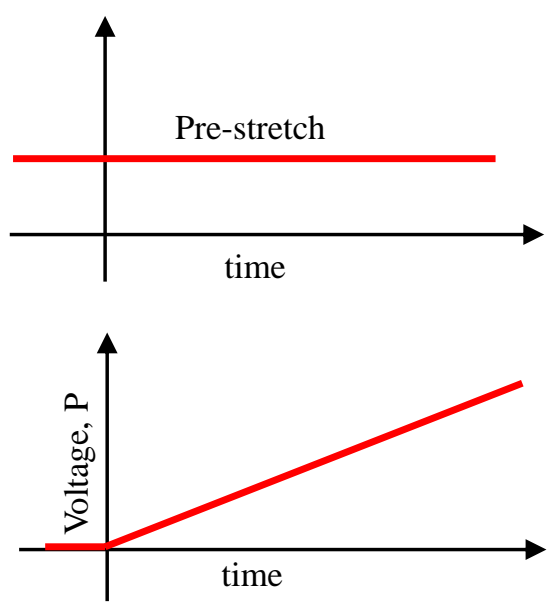

(a) Loading program

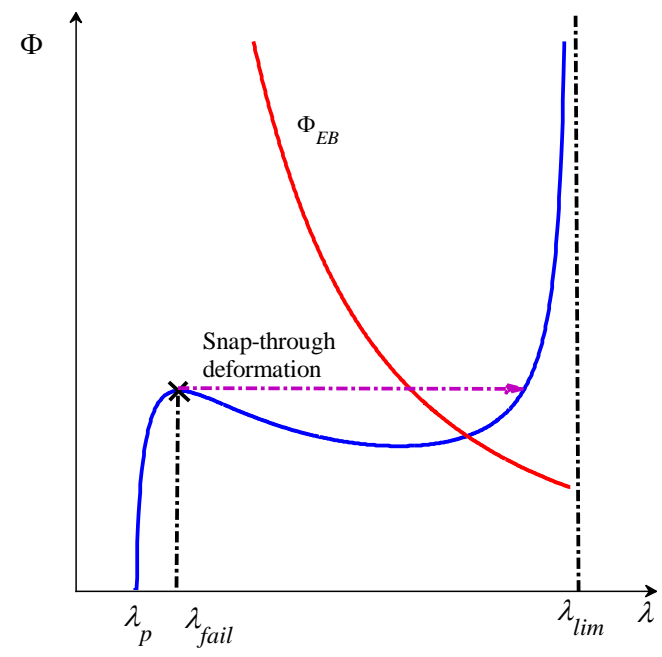

(b) Type $A_{1}$ failure mode 


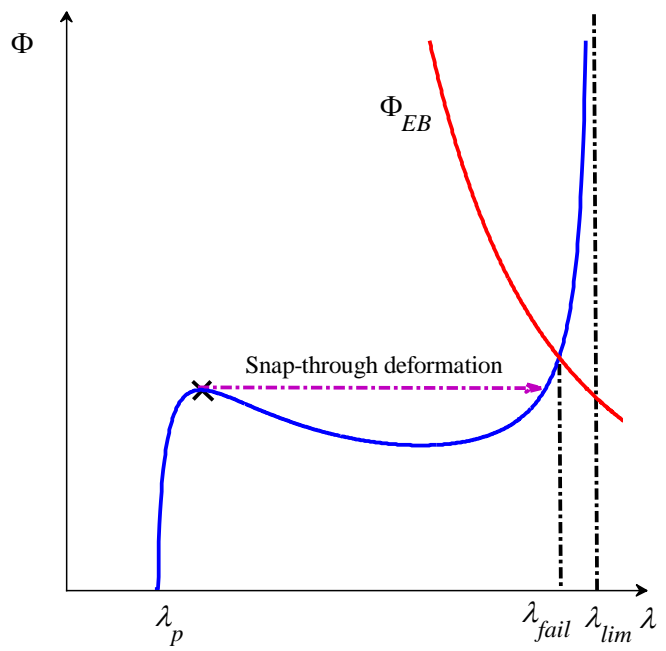

(c) Type $A_{2}$ failure mode

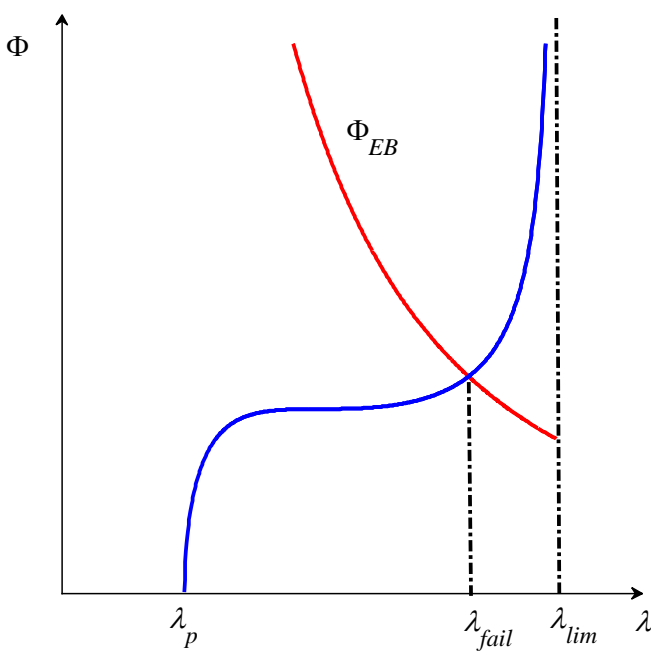

(d) Type $B$ failure mode

Figure 8. The membrane is applied with a ramping voltage after a pre-stretch (a). It may fail by: (b) EMI that leads to EB. The membrane could not survive the 'snap-through deformation' and fails when

EMI sets in. (Type $A_{1}$ failure mode); (c) EB after suffering EMI and surviving the 'snap-through deformation' (Type $A_{2}$ failure mode); (d)EB, with the EMI and 'snap-through deformation' eliminated by the pre-stretch (Type $B$ failure mode). The crosses represent the EMI points, and the horizontal dashed arrows denote the 'snap-through deformation'.

It is known from the previous section that the membrane may reach an equilibrium state without failure when it is applied with a suitable constant voltage after pre-stretch. In contrast, the membrane will inevitably fail if it is actuated by a ramping voltage (Fig. 8(a)). This is because the increasing voltage makes the true electric field $E$ surpass the electrical breakdown strength $E_{E B}=3 \times 10^{8} \mathrm{~V} / \mathrm{m}$ sooner or later, which leads to EB. Since there exists difference in the deformation results between the constant and ramping voltage actuation conditions, for the ramping voltage actuation methods, three typical failure modes after pre-stretch are specially prescribed in Fig. 8 at first for VHB $4905 / 4910$ by referring to the research of Zhao [26] and Koh [27]. In the voltage-stretch plane, Fig. 8(b) shows that a membrane with a small pre-stretch experiences EMI, which leads to EB. The horizontal dashed arrow (denoting the 'snap-through deformation') has cross with the red curve (representing the EB) in Fig. 8(b), indicating that the membrane could not exempt from EB during 
the process of the 'snap-through deformation'. Therefore, it fails when the EMI sets in, and the maximum actuation strain $\left(\left(\lambda_{\max } / \lambda_{p}-1\right) \times 100 \%\right)$ is restricted by the EMI. This kind of failure mode is classified as Type $A_{1}$ failure mode here. As shown in Fig. 8(c), a membrane with a moderate pre-stretch suffers EMI but survives the 'snap-through deformation'. Unlike the deformation of Type $A_{1}$ failure mode, the membrane can successfully jump from a small stretch to a much bigger one without suffering EB in the period of 'snap-through deformation'. Instead, it fails as EB after surviving 'snap-through deformation' as a result of the increasing voltage. Thus, the maximum actuation strain is decided by the EB after suffering EMI and surviving the 'snap-through deformation'. Type $A_{2}$ failure mode is used to categorize such kind of failure mode. Fig. 8(d) illustrates that the stretch of a membrane increases monotonically with the increasing voltage. The membrane fails as EB, with the EMI and the 'snap-through deformation' eliminated by the large pre-stretch. In this case, the maximum actuation strain of the membrane is determined by EB. Such kind of failure mode is classified as Type $B$ failure mode here.

In the following analysis, the deformations under the combined conditions of pre-stretch and ramping voltage are firstly discussed at the reference temperature $T_{0}=300 \mathrm{~K}$. Then, the relation between deformation types and the combined conditions is studied at the three representative temperatures $250 \mathrm{~K}, 300 \mathrm{~K}$ and $350 \mathrm{~K}$. Following this, the effects of factors including pre-stretch, ramping voltage and temperature on the maximum actuation strain are analyzed in detail.

In the simulation for the ramping voltage actuation condition, the ramping 
voltage is prescribed as a form of nominal electric field: $\widetilde{E}(t)=r \cdot t \mathrm{~V} / \mathrm{m}$, where $r$ is the ramping rate. By solving Eqs. (21) and (22) with initial conditions, the evolutions of the stretch $\lambda$ and the true electric field $E=\lambda^{2} \widetilde{E}$ are plotted after different pre-stretches under the conditions of two ramping rates: $r=10^{5}$ and $r=10^{3}$, respectively (Fig 9). These two ramping rates are selected to make sure that the membrane fails in a moderate time. If a large ramping rate is prescribed, the membrane would fail in a very short time, vice versa. The specific pre-stretches shown in Fig. 9 are also chosen to present the typical deformation results.

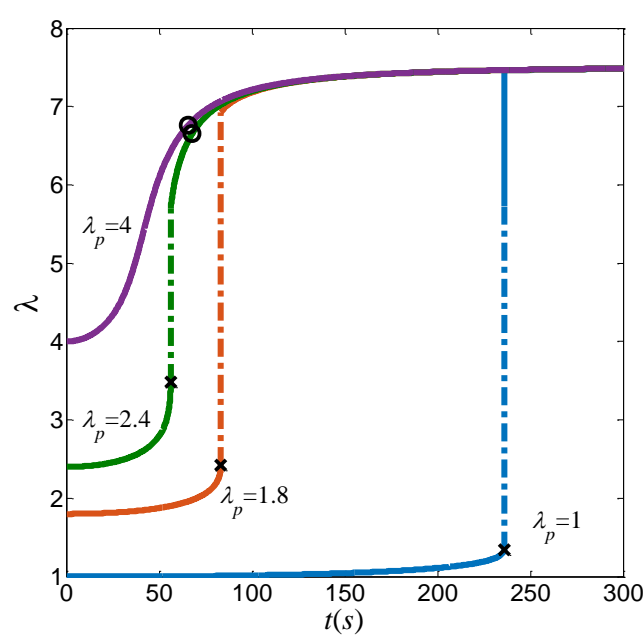

(a) $\lambda\left(r=10^{5}, T_{0}=300 \mathrm{~K}\right)$

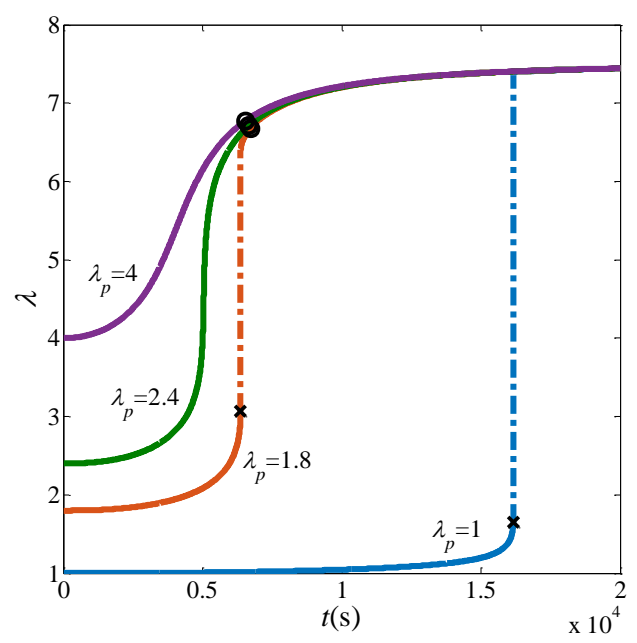

(c) $\lambda\left(r=10^{3}, T_{0}=300 \mathrm{~K}\right)$

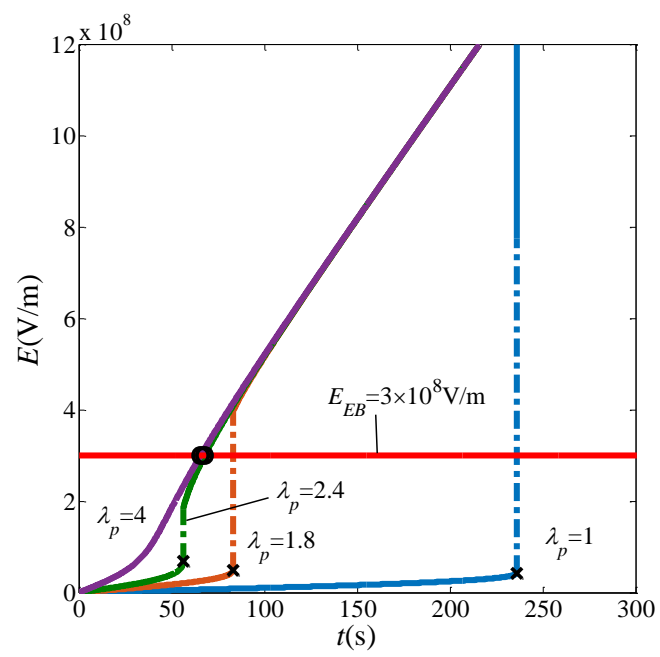

(b) $E\left(r=10^{5}, T_{0}=300 \mathrm{~K}\right)$

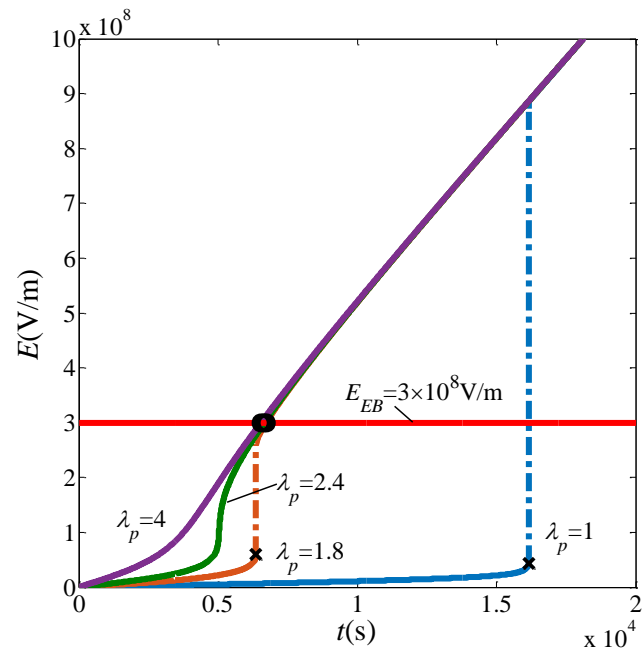

(d) $E\left(r=10^{3}, T_{0}=300 \mathrm{~K}\right)$

Figure 9. Evolutions of the stretch $\lambda$ ((a) and (c)) and the true electric field $E=\lambda^{2} \widetilde{E}$ ((b) and (d)) after different pre-stretches $\lambda_{p}$ with two specific ramping rates $r=10^{5}, r=10^{3}$. The crosses denote the 
EMI points and the circles represent the maximum stretch points. The vertical dashed lines indicate the 'snap-through deformation', and the red horizontal lines represent the electrical breakdown strength

$$
E_{E B}=3 \times 10^{8} \mathrm{~V} / \mathrm{m} \text {. }
$$

Firstly, the deformation results of membranes at the larger ramping rate $r=10^{5}$ is analyzed (Figs. 9(a) and (b)). If no pre-stretch is applied $\left(\lambda_{p}=1\right)$, the membrane suffers EMI after a period of time. Then, EB occurs as the true electric field $E$ exceeds $E_{E B}=3 \times 10^{8} \mathrm{~V} / \mathrm{m}$ during the 'snap-through deformation'. Thus, the membrane fails as Type $A_{1}$ failure mode. The maximum actuation strain under this condition is $34 \%$ $\left(\lambda_{p}=1, \lambda_{\max }=1.34\right)$. Likewise, if a small pre-stretch $\lambda_{p}=1.8$ is applied, the membrane fails as Type $A_{1}$ failure mode as well, and the corresponding maximum actuation strain is $34 \%\left(\lambda_{p}=1.8, \lambda_{\max }=2.42\right)$. As depicted in Fig. 9(b), the membrane suffers EMI but survives the 'snap-through deformation' as a moderate pre-stretch $\lambda_{p}=2.4$ is applied. The reason is that the true electric field $E$ remains below the electrical breakdown strength $E_{E B}$ during the 'snap-through deformation'. Electrical breakdown occurs as followed. In this case, the membrane fails as Type $A_{2}$ failure mode, and the maximum actuation strain is $177 \%\left(\lambda_{p}=2.4, \lambda_{\max }=6.652\right)$, which is more than 5 times as large as that of no pre-stretch condition (34\%). If a large pre-stretch $\lambda_{p}=4$ is applied, the membrane would fail as Type $B$ failure mode, with the EMI and 'snap-through deformation' eliminated. However, the corresponding maximum actuation strain in this case is only $68 \%\left(\lambda_{p}=4, \lambda_{\max }=6.758\right)$.

The deformation results under the condition of $r=10^{3}$ are similar to that of $r=10^{5}$ in general (Figs. 9(c) and (d)). However, given the same pre-stretch $\lambda_{p}=1.8$, the membrane fails as Type $A_{1}$ failure mode when the ramping rate is $r=10^{5}$, but fails as Type $A_{2}$ failure mode at the smaller ramping rate $r=10^{3}$. The corresponding 
maximum actuation strain is largely increased from $34 \%\left(r=10^{5}\right)$ to $271 \%\left(r=10^{3}\right)$. In addition, prescribed with the pre-stretch $\lambda_{p}=2.4$, the membrane fails as Type $A_{2}$ failure mode at the ramping rate $r=10^{5}$, but it fails as Type $B$ failure mode when the ramping rate is $r=10^{3}$. The maximum actuation strain slightly increases form $177 \%$ to $179 \%$ as the ramping rate decreases form $r=10^{5}$ to $r=10^{3}$. It is also indicated from the above results that the deformation failure modes and the maximum actuation strain are strongly dependent on both the pre-stretch and the ramping rate.

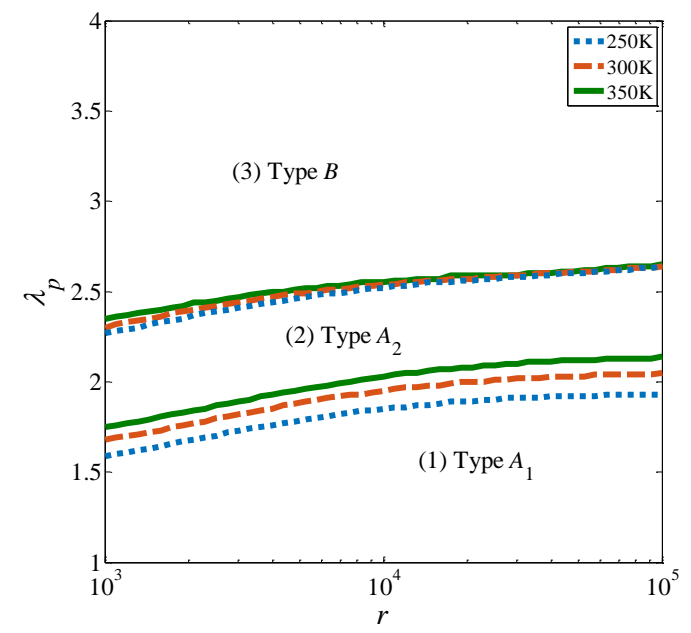

Figure 10. Phase diagram for the combined conditions of pre-stretch $\lambda_{p}$ and the ramping rate $r$ correspond to the failure modes: Type $A_{1}$, Type $A_{2}$ and Type $B$ at three representative temperatures: 250,300 and $350 \mathrm{~K}$. A point $\left(\lambda_{p}, r\right)$ on the diagram denotes a specific combined condition of pre-stretch $\lambda_{p}$ and the ramping rate $r$.

The deformation results in relation to the combined conditions of the pre-stretch $\lambda_{p}$ and the ramping rate $r$ for the three temperature conditions: $250 \mathrm{~K}, 300 \mathrm{~K}$ and $350 \mathrm{~K}$ are illustrated in Fig. 10. A point $\left(r, \lambda_{p}\right)$ in the map represents a combined condition of a ramping rate $r$ and a pre-stretch $\lambda_{p}$. The regions (1), (2) and (3) denote three different failure modes: Type $A_{1}$, Type $A_{2}$ and Type $B$, respectively. Under all temperature conditions, both the dividing lines between the region (1) and region (2) and the dividing lines between the region (2) and the region (3) decrease with the 
decrease of the ramping rate $r$. As shown in the Fig. 10, at the reference temperature $T_{0}=300 \mathrm{~K}$, the minimum pre-stretch for the membrane to fail as Type $A_{2}$ failure mode decreases from 2.05 to 1.68 as the ramping rate decreases from $r=10^{5}$ to $r=10^{3}$. Hence, a voltage with smaller ramping rate could decrease the minimum pre-stretch to help the membrane survive the EMI and 'snap-through deformation', which leads to large actuation deformation. In addition, these two kinds of dividing lines also decreases with the decreases of temperature. Thus, the minimum pre-stretch for the membrane to fail as Type $A_{2}$ failure mode and achieve large actuation deformation could be decreased by decreasing the temperature as well.

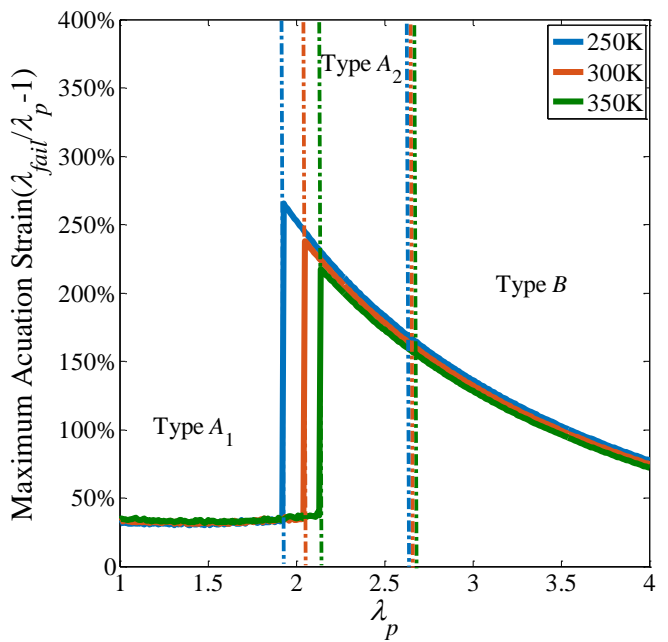

(a) $r=10^{5}$

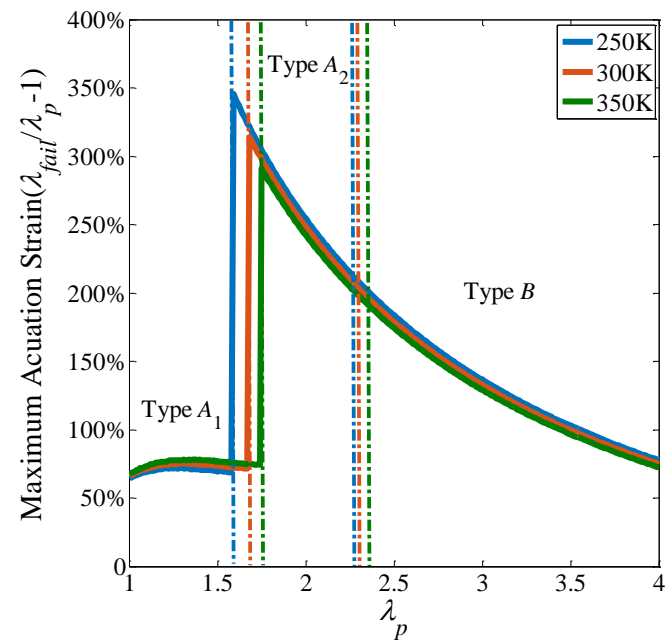

(b) $r=10^{3}$

Figure 11. Maximum actuation strain $\left(\lambda_{\max } / \lambda_{p}-1\right) \times 100 \%$ and the failure modes in relation to the pre-stretch for the two ramping rates: $r=10^{5}$ and $r=10^{3}$ at three representative temperatures: $T=250$, 300 and $350 \mathrm{~K}$. The vertical dashed lines are the dividing line between each failure type.

Figure 11 illustrates the maximum actuation strain and failure mode in relation to the pre-stretch $\lambda_{p}$ under different conditions of temperature $T$ and the ramping rate $r$. As outlined in Fig. 11, under all conditions, the membrane fails as Type $A_{1}$ failure mode when prescribed with a small pre-stretch. As a moderate or a large pre-stretch is applied, the membrane fails as Type $A_{2}$ or Type $B$ failure mode. For these two kinds 
of failure modes, the maximum actuation strain shows a decline tendency with the increasing pre-stretch in both two figures. This phenomenon could be explained by Figs. 9(a) and (c), in which the maximum stretch $\lambda_{\max }$ (denoted by the circles) is almost the same for Type $A_{2}\left(\lambda_{p}=2.4\right)$ and Type $B\left(\lambda_{p}=4\right)$ failure modes. Therefore, the maximum actuation strain $\left(\left(\lambda_{\max } / \lambda_{p}-1\right) \times 100 \%\right)$ decreases with the increase of the pre-stretch $\lambda_{p}$ for Type $A_{2}$ and Type $B$ failure modes.

Table 2. Maximum actuation strain for dielectric elastomers without pre-stretch $\left(\lambda_{p}=1\right)$.

\begin{tabular}{cccc}
\hline$r$ & $250 \mathrm{~K}$ & $300 \mathrm{~K}$ & $350 \mathrm{~K}$ \\
\hline$r=10^{5}$ & $31 \%$ & $33 \%$ & $34 \%$ \\
$r=10^{3}$ & $64 \%$ & $66 \%$ & $67 \%$ \\
\hline \hline
\end{tabular}

Table 3. The largest maximum actuation strain for dielectric elastomers with pre-stretch.

\begin{tabular}{cccc}
\hline \hline$r$ & $250 \mathrm{~K}$ & $300 \mathrm{~K}$ & $350 \mathrm{~K}$ \\
\hline$r=10^{5}$ & $265 \%\left(\lambda_{p}=1.93\right)$ & $237 \%\left(\lambda_{p}=2.05\right)$ & $217 \%\left(\lambda_{p}=2.14\right)$ \\
$r=10^{3}$ & $342 \%\left(\lambda_{p}=1.60\right)$ & $314 \%\left(\lambda_{p}=1.68\right)$ & $290 \%\left(\lambda_{p}=1.75\right)$ \\
\hline \hline
\end{tabular}

As shown in Tab. 2, under the condition of no pre-stretch $\left(\lambda_{p}=1\right)$, the maximum actuation strain slightly increases with the increase of temperature, while it remarkably increases by twofold with a decrease of the ramping rate from $r=10^{5}$ to $r=10^{3}$. Thus, a relatively small ramping rate is advised to achieve a large actuation strain for the no-pre-stretch condition. In contrast, the maximum actuation strain with pre-stretch is much larger than that of no pre-stretch condition, by comparing Tab. 3 with Tab. 2. For instance, under the conditions of $r=10^{3}$ and $T=250 \mathrm{~K}$, the maximum actuation strain without pre-stretch $\left(\lambda_{p}=1\right)$ is $64 \%$ (Tab. 2). However, provided with the same conditions of ramping rate and the temperature, the maximum actuation strain could reach up to $342 \%$ (Tab. 3) if the pre-stretch $\lambda_{p}=1.60$ is applied, which is more than five times than that of no pre-stretch condition. In summary, for the 
ramping voltage actuation deformation, the maximum actuation strain could be largely increased if a suitable pre-stretch is applied.

It is indicated from Fig. 11 that Type $A_{2}$ failure mode has the largest maximum actuation strain. Thus, for both constant and ramping voltage actuation conditions, the membrane could obtain large actuation deformation when it survives the EMI and 'snap-through deformation'. Therefore, the combined conditions of pre-stretch and the ramping rate in the region (2) of Fig. 10 that result in Type $A_{2}$ failure mode, are highly recommended in order to achieve a large actuation deformation. Then, the influences of temperature, ramping rate and pre-stretch on the maximum actuation strain are also studied in the region (2) of Fig. 10 as follows.

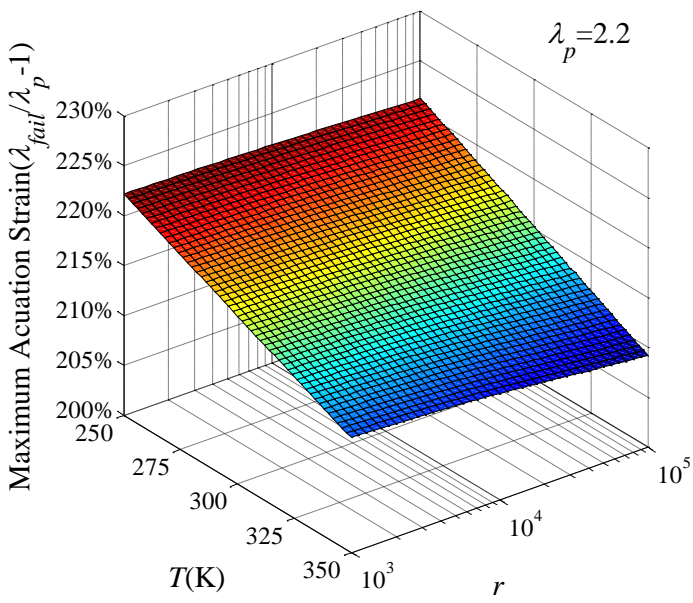

(a)

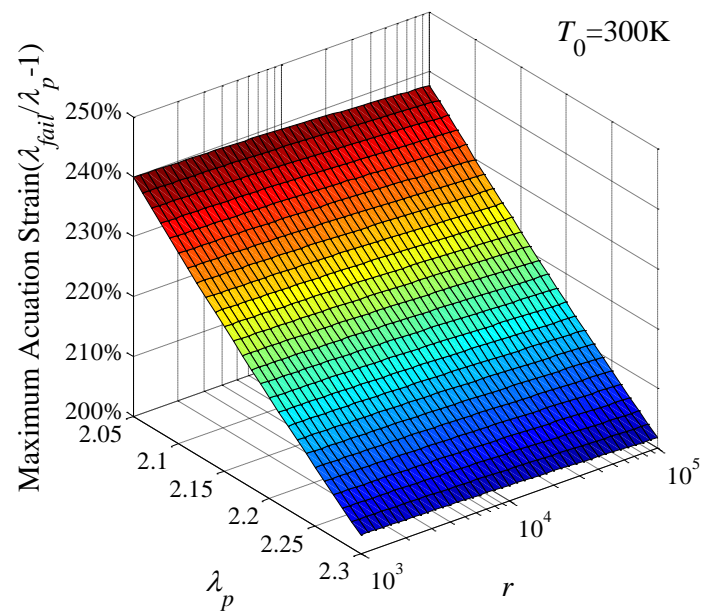

(b)

Figure 12. Maximum actuation strain for dielectric elastomers with Type $A_{2}$ failure mode: (a) pre-stretch is fixed as $\lambda_{p}=2.2$. (b) temperature is prescribed with $T=300 \mathrm{~K}$.

As all the three factors: pre-stretch $\lambda_{p}$, ramping rate $r$ and temperature $T$ have influences on the maximum actuation strain of Type $A_{2}$ failure mode, pre-stretch and temperature are separately fixed in order to study the effects of other two factors. Firstly, $\lambda_{p}=2.2$ is selected to make sure within the temperature range $T=250 \mathrm{~K} \sim 300 \mathrm{~K}$ and the ramping range $r=10^{3} \sim 10^{5}$, the membrane could fail as Type $A_{2}$ failure mode. 
As shown in Fig. 12(a), when the pre-stretch is given as $\lambda_{p}=2.2$, the maximum actuation strain of the dielectric elastomer shows an increasing trend with the decrease of temperature. On the other hand, as the ramping rate $r$ decreases from $10^{5}$ to $10^{3}$, the maximum actuation strain experiences a slight increase. If the temperature condition is fixed as $T_{0}=300 \mathrm{~K}$ (Fig. 12(b)), the maximum actuation strain increases with a decrease of pre-stretch. In conclusion, under the best combined conditions of pre-stretch and the ramping rate, the maximum actuation strain increases with the decrease of pre-stretch, ramping rate and temperature. Thus, for the ramping voltage actuation deformation, the combined conditions of a relatively small pre-stretch, small ramping rate and low temperature, which helps the membrane survive the EMI and 'snap-through' and fail as Type $A_{2}$ failure mode, are recommended for the realization of a large actuation deformation.

\section{Conclusion}

In this study, with the consideration of viscoelasticity and temperature, the equation of state for the dissipative elastomer: VHB 4905/4910 is obtained under the guidance of nonequilibrium thermodynamics. For the first time, the maximum actuation strain of dissipative dielectric elastomers with consideration of simultaneous effect of temperature and pre-stretch is studied under the conditions of the constant and ramping voltage actuations. The results show that the pre-stretch does not always ensure the improvement of maximum actuation strain, and the optimization of conditions of pre-stretch, voltage and temperature is crucial for the dielectric 
elastomers to achieve large actuation deformation. For both the constant and ramping voltage conditions, the large actuation strain could be obtained if the membrane could survive the EMI and 'snap-through deformation'. In the case of constant voltage actuation deformation, the maximum actuation strain increases with the increase of the constant voltage, but decreases with the increase of pre-stretch and temperature in the area of the best combined conditions. Regarding to the ramping voltage condition, the maximum actuation strain increases with the decrease of pre-stretch, ramping rate and temperature within the best combined conditions areas. The mathematical model established in this work may offer a great help in the design of the high performance dielectric elastomer actuators.

\section{Acknowledgements}

The project is supported by China Postdoctoral Science Foundation. The authors also wish to thank the support by the Science Fund of State Key Laboratory of Advanced Design and Manufacturing for Vehicle Body (Grant No. 51375001), Research Project of State Key Laboratory of Mechanical Systems and Vibration MSV201613, Research Project of Guangxi Provincial Department of Science and Technology (Grant No. 1598008-18), and Research Project of Key Laboratory of Advanced Manufacture Technology for Automobile Parts (2014KLMT04).

\section{Reference}

[1] Y. Bar-Cohen, Electroactive polymers as artificial muscles-capabilities, potentials and challenges, Handbook on biomimetics, 8 (2000).

[2] J.D. Madden, N. Vandesteeg, P. Anquetil, P.G. Madden, A. Takshi, R.Z. Pytel, S.R. 
Lafontaine, P. Wieringa, I.W. Hunter, Artificial muscle technology: physical principles and naval prospects, Oceanic Engineering, IEEE Journal of, 29 (2004) 706-728.

[3] E. Biddiss, T. Chau, Dielectric elastomers as actuators for upper limb prosthetics: Challenges and opportunities, Medical engineering \& physics, 30 (2008) 403-418.

[4] R. Pelrine, R.D. Kornbluh, J. Eckerle, P. Jeuck, S. Oh, Q. Pei, S. Stanford, Dielectric elastomers: Generator mode fundamentals and applications, in: SPIE's 8th Annual International Symposium on Smart Structures and Materials, International Society for Optics and Photonics, 2001, pp. 148-156.

[5] Y. Bar-Cohen, Electroactive polymers for refreshable Braille displays, SPIE Newsroom, 11 (2009).

[6] Y. Bar-Cohen, Electroactive polymers as artificial muscles capabilities - Potentials and challenges, Robotics 2000, Proceedings, (2000) 188-196.

[7] A. O'Halloran, F. O'Malley, P. McHugh, A review on dielectric elastomer actuators, technology, applications, and challenges, J Appl Phys, 104 (2008).

[8] R. Pelrine, R. Kornbluh, Q.B. Pei, S. Stanford, S.J. Oh, J. Eckerle, R. Full, M. Rosenthal, K. Meijer, Dielectric elastomer artificial muscle actuators: Toward biomimetic motion, Smart Structures and Materials 2002: Electroactive Polymer Actuators and Devices (Eapad), 4695 (2002) 126-137.

[9] R. Pelrine, R. Kornbluh, J. Eckerle, P. Jeuck, S.J. Oh, Q.B. Pei, S. Stanford, Dielectric elastomers: Generator mode fundamentals and applications, Smart Structures and Materials 2001: Electroactive Polymer Actuators and Devices, 4329 (2001) 148-156.

[10] Z.G. Suo, Theory of Dielectric Elastomers, Acta Mech Solida Sin, 23 (2010) 549-578.

[11] F. Carpi, D. De Rossi, R. Kornbluh, R.E. Pelrine, P. Sommer-Larsen, Dielectric elastomers as electromechanical transducers: Fundamentals, materials, devices, models and applications of an emerging electroactive polymer technology, Elsevier, 2011.

[12] R. Pelrine, R. Kornbluh, Q. Pei, J. Joseph, High-speed electrically actuated elastomers with strain greater than 100\%, Science, 287 (2000) 836-839.

[13] W. Sun, H. Wang, J. Zhou, Actuation and instability of interconnected dielectric elastomer balloons, Applied Physics A, 119 (2015) 443-449.

[14] B. Li, H. Chen, J. Qiang, S. Hu, Z. Zhu, Y. Wang, Effect of mechanical pre-stretch on the stabilization of dielectric elastomer actuation, Journal of Physics D: Applied Physics, 44 (2011) 155301.

[15] B. Li, L. Liu, Z. Suo, Extension limit, polarization saturation, and snap-through instability of dielectric elastomers, International Journal of Smart and Nano Materials, 2 (2011) 59-67.

[16] K. Stark, C. Garton, Electric strength of irradiated polythene, (1955).

[17] M.T. Ghoneim, A. Kutbee, F. Ghodsi Nasseri, G. Bersuker, M. Hussain, Mechanical anomaly impact on metal-oxide-semiconductor capacitors on flexible silicon fabric, Applied Physics Letters, 104 (2014) 234104.

[18] M. Ieda, Dielectric breakdown process of polymers, IEEE Transactions on Electrical Insulation, (1980) 206-224. 
[19] M.T. Ghoneim, J.P. Rojas, C.D. Young, G. Bersuker, M.M. Hussain, Electrical analysis of high dielectric constant insulator and metal gate metal oxide semiconductor capacitors on flexible bulk mono-crystalline silicon, IEEE Transactions on Reliability, 64 (2015) 579-585.

[20] X.H. Zhao, W. Hong, Z.G. Suo, Electromechanical hysteresis and coexistent states in dielectric elastomers, Phys Rev B, 76 (2007).

[21] X. Zhao, Z. Suo, Method to analyze electromechanical stability of dielectric elastomers, Applied Physics Letters, 91 (2007) 061921.

[22] S.M. Ha, W. Yuan, Q. Pei, R. Pelrine, S. Stanford, Interpenetrating Polymer Networks for High - Performance Electroelastomer Artificial Muscles, Advanced Materials, 18 (2006) 887-891.

[23] Z. Suo, J. Zhu, Dielectric elastomers of interpenetrating networks, Applied Physics Letters, 95 (2009) 232909.

[24] R. Shankar, T.K. Ghosh, R.J. Spontak, Electroactive nanostructured polymers as tunable actuators, Advanced Materials, 19 (2007) 2218-2223.

[25] C. Keplinger, M. Kaltenbrunner, N. Arnold, S. Bauer, Röntgen's electrode-free elastomer actuators without electromechanical pull-in instability, Proceedings of the National Academy of Sciences, 107 (2010) 4505-4510.

[26] X. Zhao, Z. Suo, Theory of dielectric elastomers capable of giant deformation of actuation, Physical Review Letters, 104 (2010) 178302.

[27] S.J.A. Koh, T. Li, J. Zhou, X. Zhao, W. Hong, J. Zhu, Z. Suo, Mechanisms of large actuation strain in dielectric elastomers, Journal of Polymer Science Part B: Polymer Physics, 49 (2011) 504-515.

[28] C. Keplinger, T. Li, R. Baumgartner, Z. Suo, S. Bauer, Harnessing snap-through instability in soft dielectrics to achieve giant voltage-triggered deformation, Soft Matter, 8 (2012) 285-288.

[29] R. Palakodeti, M. Kessler, Influence of frequency and prestrain on the mechanical efficiency of dielectric electroactive polymer actuators, Materials Letters, 60 (2006) 3437-3440.

[30] J.-S. Plante, S. Dubowsky, On the performance mechanisms of dielectric elastomer actuators, Sensors and Actuators A: Physical, 137 (2007) 96-109.

[31] J.-S. Plante, S. Dubowsky, Large-scale failure modes of dielectric elastomer actuators, International journal of solids and structures, 43 (2006) 7727-7751.

[32] P. Lochmatter, G. Kovacs, M. Wissler, Characterization of dielectric elastomer actuators based on a visco-hyperelastic film model, Smart Materials and Structures, 16 (2007) 477.

[33] C. Keplinger, M. Kaltenbrunner, N. Arnold, S. Bauer, Capacitive extensometry for transient strain analysis of dielectric elastomer actuators, Applied Physics Letters, 92 (2008) 192903.

[34] J. Zhang, B. Li, H. Chen, Q. Pei, Dissipative performance of dielectric elastomers under various voltage waveforms, Soft matter, 12 (2016) 2348-2356.

[35] H.S. Park, T.D. Nguyen, Viscoelastic effects on electromechanical instabilities in dielectric elastomers, Soft Matter, 9 (2013) 1031-1042.

[36] J. Zhang, H. Chen, L. Tang, B. Li, J. Sheng, L. Liu, Modelling of spring roll 
actuators based on viscoelastic dielectric elastomers, Applied Physics A, 119 (2015) 825-835.

[37] J. Sheng, H. Chen, B. Li, Effect of temperature on the stability of dielectric elastomers, Journal of Physics D: Applied Physics, 44 (2011) 365406.

[38] L. Liu, Y. Liu, K. Yu, J. Leng, Thermoelectromechanical stability of dielectric elastomers undergoing temperature variation, Mechanics of Materials, 72 (2014) 33-45.

[39] J. Sheng, H. Chen, B. Li, Y. Wang, Influence of the temperature and deformation - dependent dielectric constant on the stability of dielectric elastomers, Journal of Applied Polymer Science, 128 (2013) 2402-2407.

[40] A. Fortino, J. Biermann, U. Bakirdögen, M. Reke, Active vibration damping with electro-active polymers using state feedback control.

[41] J. Sheng, H. Chen, B. Li, L. Chang, Temperature dependence of the dielectric constant of acrylic dielectric elastomer, Applied Physics A, 110 (2013) 511-515.

[42] C. Jean-Mistral, A. Sylvestre, S. Basrour, J. Chaillout, Dielectric properties of polyacrylate thick films used in sensors and actuators, Smart materials and structures, 19 (2010) 075019.

[43] C.C. Foo, S.J.A. Koh, C. Keplinger, R. Kaltseis, S. Bauer, Z. Suo, Performance of dissipative dielectric elastomer generators, Journal of Applied Physics, 111 (2012) 094107.

[44] X.H. Zhao, S.J.A. Koh, Z.G. Suo, Nonequilibrium Thermodynamics of Dielectric Elastomers, Int J Appl Mech, 3 (2011) 203-217.

[45] W. Hong, Modeling viscoelastic dielectrics, J Mech Phys Solids, 59 (2011) 637-650.

[46] C.C. Foo, S.J.A. Koh, C. Keplinger, R. Kaltseis, S. Bauer, Z.G. Suo, Performance of dissipative dielectric elastomer generators, J Appl Phys, 111 (2012).

[47] C.C. Foo, S.Q. Cai, S.J.A. Koh, S. Bauer, Z.G. Suo, Model of dissipative dielectric elastomers, J Appl Phys, 111 (2012).

[48] Z. Suo, Theory of dielectric elastomers, Acta Mechanica Solida Sinica, 23 (2010) 549-578.

[49] L.W. Liu, Y.J. Liu, B. Li, K. Yang, T.F. Li, J.S. Leng, Thermo-electro-mechanical instability of dielectric elastomers, Smart Mater Struct, 20 (2011).

[50] J.J. Sheng, H.L. Chen, B. Li, Effect of temperature on the stability of dielectric elastomers, J Phys D Appl Phys, 44 (2011).

[51] C.O. Horgan, G. Saccomandi, Finite thermoelasticity with limiting chain extensibility, J Mech Phys Solids, 51 (2003) 1127-1146.

[52] E. Bilgili, B. Bernstein, H. Arastoopour, Inhomogeneous shearing deformation of a rubber-like slab within the context of finite thermoelasticity with entropic origin for the stress, Int J Nonlinear Mech, 36 (2001) 887-900.

[53] A. Gent, A new constitutive relation for rubber, Rubber chemistry and technology, 69 (1996) 59-61.

[54] C.C. Foo, S. Cai, S.J.A. Koh, S. Bauer, Z. Suo, Model of dissipative dielectric elastomers, Journal of Applied Physics, 111 (2012) 034102.

[55] X. Zhao, S.J.A. Koh, Z. Suo, Nonequilibrium thermodynamics of dielectric 
elastomers, International journal of applied mechanics, 3 (2011) 203-217.

[56] S. Michel, X.Q. Zhang, M. Wissler, C. Löwe, G. Kovacs, A comparison between silicone and acrylic elastomers as dielectric materials in electroactive polymer actuators, Polymer international, 59 (2010) 391-399.

[57] S.J.A. Koh, X. Zhao, Z. Suo, Maximal energy that can be converted by a dielectric elastomer generator, Applied Physics Letters, 94 (2009) 262902.

[58] L.A. Dissado, J.C. Fothergill, Electrical degradation and breakdown in polymers, IET, 1992.

[59] X. Zhao, W. Hong, Z. Suo, Electromechanical hysteresis and coexistent states in dielectric elastomers, Physical review B, 76 (2007) 134113.

[60] Z. Suo, Mechanics of stretchable electronics and soft machines, Mrs Bulletin, 37 (2012) 218-225. 